\title{
Strain Engraftment Competition and Functional Augmentation in a Multi-donor Fecal Microbiota Transplantation Trial for Obesity
}

\section{Brooke Wilson}

Liggins Institute, University of Auckland

\section{Tommi Vatanen}

Liggins Institute, University of Auckland

Thilini Jayasinghe

Liggins Institute, University of Auckland

Karen Leong

Liggins Institute, University of Auckland

José Derraik

Liggins Institute, University of Auckland

\section{Benjamin Albert}

Liggins Institute, University of Auckland

Valentina Chiavaroli

Liggins Institute, University of Auckland

\section{Darren Svirskis}

Liggins Institute, University of Auckland

\section{Kathryn Beck}

Massey University College of Health

\section{Cathryn Conlon}

Massey University College of Health

\section{Yannan Jiang}

The University of Auckland Department of Statistics

\section{William Schierding}

Liggins Institute, University of Auckland

\section{David Holland}

Counties Manukau DHB: Counties Manukau District Health Board

\section{Wayne Cutfield}

Liggins Institute, University of Auckland

Justin Martin O'Sullivan ( $\nabla$ justin.osullivan@auckland.ac.nz )

The Liggins Institute, University of Auckland, New Zealand https://orcid.org/0000-0003-2927-450X 
Research

Keywords: Donor selection, fecal microbiota transplantation (FMT), Prevotella to Bacteroides (P/B) ratio

Posted Date: October 28th, 2020

DOI: https://doi.org/10.21203/rs.3.rs-97360/v1

License: (c) (i) This work is licensed under a Creative Commons Attribution 4.0 International License. Read Full License

Version of Record: A version of this preprint was published at Microbiome on May 13th, 2021. See the published version at https://doi.org/10.1186/s40168-021-01060-7. 


\section{Strain engraftment competition and functional augmentation in a multi-}

\section{2 donor fecal microbiota transplantation trial for obesity}

3 Brooke C. Wilson ${ }^{1}$, Tommi Vatanen ${ }^{1,2}$, Thilini N. Jayasinghe ${ }^{1}$, Karen S. W. Leong ${ }^{1,3}$, José G. B.

4 Derraik $^{1,3}$, Benjamin B. Albert ${ }^{1,3}$, Valentina Chiavaroli ${ }^{1}$, Darren M. Svirskis ${ }^{4}$, Kathryn L. Beck ${ }^{5}$, Cathryn

5 A. Conlon ${ }^{5}$, Yannan Jiang ${ }^{6}$, William Schierding ${ }^{1}$, David J. Holland ${ }^{7}$, Wayne S. Cutfield ${ }^{1,3^{*}}$, Justin M.

6 O'Sullivan ${ }^{1,3^{*}}$

$7 \quad$ The Liggins Institute, University of Auckland, New Zealand

${ }^{2}$ The Broad Institute of MIT and Harvard, Cambridge, MA, USA

${ }^{3}$ A Better Start - National Science Challenge, Auckland, New Zealand

${ }^{4}$ School of Pharmacy, Faculty of Medical and Health Sciences, University of Auckland, New Zealand

${ }^{5}$ School of Sport, Exercise and Nutrition, College of Health, Massey University, New Zealand

${ }^{6}$ Department of Statistics, University of Auckland, New Zealand

${ }^{7}$ Department of Infectious Diseases, Counties Manukau District Health Board, Auckland, New Zealand

*Joint correspondence: Wayne S. Cutfield w.cutfield@auckland.ac.nz and Justin M. O'Sullivan justin.osullivan@auckland.ac.nz

\section{Authors email addresses}

Brooke C. Wilson b.wilson@auckland.ac.nz; Tommi Vatanen t.vatanen@auckland.ac.nz; Thilini N. Jayasinghe tmad109@aucklanduni.ac.nz; Karen S.W. Leong k.leong@auckland.ac.nz; José G. B. Derraik j.derraik@auckland.ac.nz; Benjamin B. Albert b.albert@auckland.ac.nz; Valentina Chiavaroli valentinachiavaroli@hotmail.it; Darren M. Svirskis d.svirskis@auckland.ac.nz; Kathryn L. Beck

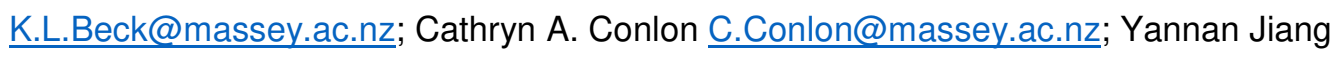
y.jiang@auckland.ac.nz; William Schierding w.schierding@auckland.ac.nz; David J. Holland 


\section{ABSTRACT}

\section{Background}

Donor selection is an important factor influencing the engraftment and efficacy of fecal microbiota transplantation (FMT) for complex conditions associated with microbial dysbiosis. However, the degree, variation, and stability of strain engraftment has not yet been assessed in the context of multiple donors.

Methods

We conducted a double-blinded randomised control trial of FMT in 42 adolescents with obesity. Participants were randomised to receive multi-donor FMT (capsules containing the fecal microbiota of four sex-matched lean donors) or placebo (saline capsules). Following a bowel cleanse, participants ingested a total of 28 capsules over two consecutive days. Capsules from individual donors and participant stool samples collected at baseline, 6-, 12-, and 26-weeks post-treatment were analysed by shotgun metagenomic sequencing allowing us to track bacterial strain engraftment and its functional implications on recipients' gut microbiomes.

\section{Results}

Multi-donor FMT sustainably altered the structure and the function of the gut microbiome. In what was effectively a microbiome competition experiment, we discovered that two donor microbiomes (one female, one male) dominated strain engraftment and were characterised by high microbial diversity and a high Prevotella to Bacteroides (P/B) ratio. Engrafted strains led to enterotype-level shifts in community composition and provided genes that altered the metabolic potential of the community. Despite our attempts to standardise FMT dose and origin, FMT recipients varied widely in their engraftment of donor strains.

\section{Conclusion}

51 Our study provides evidence for the existence of FMT super-donors whose microbiomes are highly effective at engrafting in the recipient gut. Dominant engrafting male and female donor microbiomes 
harboured diverse microbial species and genes, and were characterised by a high P/B ratio. Yet, the high variability of strain engraftment among FMT recipients suggests the host environment also plays a critical role in mediating FMT receptivity.

\section{Trial registration}

The Gut Bugs Trial was registered with the Australian New Zealand Clinical Trials Registry (ACTRN12615001351505)

\section{Trial protocol}

The trial protocol is available at https://bmjopen.bmj.com/content/9/4/e026174.long

\section{BACKGROUND}

Fecal microbiota transplantation (FMT) is currently being investigated for its efficacy in treating a variety of chronic disorders in which the gut microbiome is presumably implicated. Among these, obesity and metabolic disorders have been a key focus, given their global health burden and lack of effective treatment options (1). Pioneering experiments in mice revealed that obese and lean phenotypes could be transferred through the fecal microbiota of human donors (2-4). Clinical trials of FMT have been comparatively less impressive with respect to weight loss in individuals with obesity (5-9). Nonetheless, FMT has been shown to transiently improve insulin sensitivity in some individuals $(8,9)$, and alter fat distribution in others (Leong et al., in submission). While these studies point to a complex relationship between the human gut microbiota and metabolic health, the mechanisms and levels of strain engraftment required to elicit these effects remain poorly characterized.

Efficient engraftment of donor strains is likely a prerequisite for functional alteration of the host's gut microbiome (10). Several studies trialling FMT for inflammatory bowel disease (IBD) have reported greater clinical response in recipients whose gut microbiomes are more receptive to FMT engraftment (11-15). Similarly, the composition of the donor's gut microbiota has also been shown to influence FMT outcomes $(11,12,16)$. The idea that some stool is therapeutically better than others led to the concept 
of "super-donors" (10), and has prompted researchers to trial multi-donor FMT approaches (17), or employ more rationalized donor selection based on perceived microbiota fitness (18). Multi-donor FMT involves the administration of fecal microbiota from multiple donors, with the goal of homogenising donor-specific effects and increasing microbial diversity (19).

Replacing a disease-associated microbiome with a one-off dose of donor microbiota is not trivial, and presumably involves a variety of competitive interactions between the endogenous and exogenous communities, restrained within the niche environment supplied by the host. Strain-resolved metagenomics have been instrumental in improving our understanding of FMT engraftment, revealing that donor strains can stably coexist with recipient's endogenous strains for months after FMT $(20,21)$. However, the picture is far from complete, and the dynamics and functional implications of strain engraftment in the context of multiple donor sources is yet to be investigated.

To address these gaps, we metagenomically profiled stool samples from healthy, lean donors and adolescents with obesity participating in a double-blinded randomized-controlled trial for FMT. Fecal microbiota was harvested from multiple donors and administered over two consecutive days in a capsule form that enabled competition between the individual microbiomes. Recipients' gut microbiomes were tracked for up to 6 months post-treatment. Specifically, we sought to quantify the degree of donor strain engraftment, uncover any donor-specific trends, track the persistence of donor strains, and identify any functional effects of strain engraftment on the host's microbial community.

\section{METHODS}

\section{Study design and participant information}

The Gut Bugs Trial was a double-blinded, randomized, placebo-controlled trial that tested the efficacy of FMT capsules to treat adolescents with obesity (35). This study was approved by the Northern A Health and Disability Ethics Committee on 8th November 2016 (16/NTA/172). A total of 87 post-pubertal adolescents with obesity participated in the trial (51 females, 36 males, aged $14-18$ years, BMI 37.7 $\pm 5.3 \mathrm{~kg} / \mathrm{m}^{2}$, total body fat $47.5 \pm 5.6 \%$ ). Nine donors ( 4 females, 5 males, aged $19-27$ years, BMI 22.7 $\pm 1.9 \mathrm{~kg} / \mathrm{m}^{2}$, total body fat $18.4 \pm 3.2 \%$ ) were selected following extensive health and pathogen screening as documented in the published trial protocol (35). 
Recipients were stratified by sex and randomized $1: 1$ to receive 28 acid-resistant capsules over two consecutive days containing either the active treatment (multi-donor FMT) or placebo (saline solution). The fecal microbiota used for FMT capsules was derived from four healthy same-sex lean donors. To standardize treatment, the same donors were used for the entire course of the study with the exception of one male donor, DM05, who was replaced after the first male treatment round with DM12 due to illness.

The day before treatment, recipients ingested a GlycoPrep-C solution (Fresenius Kabi, New Zealand) to cleanse the bowel and reduce the endogenous microbial load. Participants were instructed to maintain their usual diet and physical activity patterns throughout the trial. Clinical assessments were conducted at baseline, 6-weeks, 12-weeks, and 26-weeks post-treatment (35).

\section{Fecal microbiota transplantation procedure}

Fecal microbiota was harvested from fresh donor stool and double encapsulated using a validated protocol (Youngster et al., 2014). Capsules were prepared using acid-resistant DR Caps ${ }^{\mathrm{TM}}$ (Capsugel, USA) which are specifically designed to release their contents in the proximal bowel. Each capsule contained $0.25 \mathrm{~g}$ of microbiota suspended in $0.5 \mathrm{ml}$ cryoprotective saline solution $(0.9 \% \mathrm{NaCl}, 15 \%$ glycerol). Placebo capsules were visually indistinguishable and contained $0.5 \mathrm{ml}$ of sterile saline solution $\left(0.9 \% \mathrm{NaCl}, 15 \%\right.$ glycerol). Capsules were stored at $-80^{\circ} \mathrm{C}$ for approximately one week before administration.

Due to the nature of recruitment, participants were treated in batches, with fresh FMT capsules prepared for each treatment batch. In total, there were 8 female and 6 male treatment batches. Following a bowel cleanse and overnight fast, participants received their allocated capsules. FMT recipients received 7 capsules from each of the 4 same-sex donor, for a combined dose of 28 capsules; 16 capsules on the first day and 12 capsules the following day. Capsules were swallowed with a glass of water under clinical supervision. 
Fresh stool samples were collected on site from participants at baseline (prior to bowel cleansing and treatment), as well as at 6-weeks, 12-weeks, and 26-weeks post-treatment. A $200 \mathrm{mg}$ aliquot was taken from the middle-section of the stool and transferred to a $2 \mathrm{ml}$ LoBind DNA tube for temporary storage at $-80^{\circ} \mathrm{C}$. In addition, FMT capsules from each batch of donations were reserved for microbiome assessment. Nucleic acid extraction was performed within five days of stool collection using a modified protocol of the AllPrep DNA/RNA Mini Kit (Qiagen, USA) (Giannoukos et al., 2012). Firstly, stool aliquots were incubated in $100 \mu \mathrm{l}$ of lysis buffer (30 mM Tris-HCl, $1 \mathrm{mM}$ EDTA, $15 \mathrm{mg} / \mathrm{ml}$ lysozyme) for 10 minutes at room temperature with regular agitation. Samples were then mixed with $1.2 \mathrm{ml} R L T$ plus buffer (Qiagen, USA), $12 \mu$ l beta-mercaptoethanol (Sigma-Aldrich, USA), and $1 \mathrm{ml}$ of acid washed glass beads ( $\leq 106 \mu \mathrm{m},-140$ U.S. sieve; Sigma Aldrich, USA) and shaken vigorously at $30 \mathrm{~Hz}$ for 10 minutes on a TissueLyser II (Qiagen, USA). The homogenate was then passed through a QIAshredder spin column (Qiagen, USA), before continuing on with the standard AllPrep DNA/RNA Mini kit protocol (Qiagen, USA) eluting in $100 \mu$ l of EB buffer. DNA purity was assessed using a NanoPhotometer N60 (Implen GmbH, Germany) and quantified by Qubit dsDNA Broad Range assay (Thermo Fisher Scientific, USA).

\section{Metagenomic library preparation and sequencing}

A total of 381 stool samples from recipients and donors were analysed by shotgun metagenomic sequencing. Metagenomic sequencing libraries were prepared using the NEBNext Ultra DNA Library Prep Kit for Illumina (NEB, USA) following the manufacturer's protocol. In brief, DNA was fragmented by sonication to an average size of $300 \mathrm{bp}$, and resulting fragments were end-polished, A-tailed, and ligated with sequence adaptors. Following PCR amplification, DNA fragments were purified (AMPure SP system, Beckman Coulter, USA), assessed for size distribution (Agilent2100 Bioanalyzer, USA) and quantified by real-time PCR. Clusters were generated using the cBot Cluster Generation System, and sequencing was performed on an Illumina NovaSeq6000 platform, generating an average of 23 million reads per sample (150 bp paired-end reads). Raw sequencing files were processed with bioBakery workflows using docker images available at http://huttenhower.sph.harvard.edu/biobakery workflows. 
followed by removal of low-quality reads and human sequences with KneadData. Post-processed metagenomic sequencing files and accompanying metadata are deposited in NCBl's SRA database (BioProject PRJNA637785).

\section{Taxonomic profiling}

Species-level taxonomic profiles were generated by MetaPhIAn2 v2.7.7 (22). Metaphlan2 uses read coverage of clade-specific marker genes to estimate the relative abundance of taxonomic clades present within a sample. Taxonomic profiles included bacteria, archaea, viruses, and eukaryotic microbes. Shannon's diversity index was used to estimate alpha diversity, and Bray-Curtis dissimilarity was used to estimate beta diversity at the bacterial species-level.

Differences in the overall structure of the gut microbiome, based on Bray-Curtis dissimilarities, were assessed by permutational multivariate analysis of variance (PERMANOVA) using the adonis2 function in the vegan $\mathrm{R}$ package (27). The effect of treatment group was assessed cross-sectionally at each time point, with marginal adjustments for sequence batch, sex, age, ethnicity, and antibiotic usage (10,000 permutations). Nominal $p$-values from PERMANOVA were adjusted for multiple testing using Benjamini-Hochberg procedure to obtain $q$-values and results with $\mathrm{q}<0.1$ were considered statistically significant.

Associations between individual species and metadata were examined using general linear models as implemented in the MaAsLin2 $\mathrm{R}$ package (28). Species relative abundance profiles were logtransformed, and species had to be present in at least $10 \%$ of samples to be included in analyses. For assessing the effect of treatment from baseline to week 6 on species relative abundance, time point was used as a fixed effect variable, with participant ID added as a random effect, including each treatment group in turn (i.e. FMT and placebo profiles run separately). Differential species associated with FMT were visualized by pheatmap (29), with manual clustering according to the significance level and direction of association. 


\section{Strain inference and engraftment analysis}

190 Profiling of the dominant strain of a given species was achieved by SNP-based haplotyping using StrainPhIAn (24), requiring a minimum coverage of 5 bases for SNP calling (min_read_depth 5), and adding the option "--relaxed_parameters3". To estimate phylogenetic relationships between conspecific strains from different individuals, DNA similarity distances were calculated for SNP haplotypes using the Jukes and Cantor (JC69) model, as implemented in the phangorn R package (36). An initial tree was constructed using Unweighted Pair Group Method with Arithmetic Mean (UPGMA) hierarchical clustering, which was optimized by maximum likelihood estimation using the Kimura model (K80). Phylogenetic trees were visualized using the ggtree $\mathrm{R}$ package (31). To systematically quantify strain engraftment events for all profiled species, DNA distances were normalized by the median distance across all pairwise comparisons of a given species. This enabled us to account for the varying degrees of strain diversity across species. We selected a conservative normalized DNA distance threshold of 0.2 such that any strains with higher similarity were deemed identical (Fig. 3B).

\section{Functional profiling}

204

Functional profiling was performed by HUMAnN2 v0.11.2 (23), which involved mapping post-processed reads against the pangenomes of detected species, allowing read-count-based quantification of the microbial gene families present within each sample. Identified gene families were further mapped to the MetaCyc database to provide quantification of metabolic pathways. Both gene family and pathway profiles were stratified by contributing organisms. For each sample, gene richness was calculated by counting the number of unique gene families present. When comparing gene richness of donors and participants at baseline, multiple samples from each donor, corresponding to each donation batch, were averaged. Associations between individual pathways and treatment were assessed cross-sectionally for each time point with general linear models using MaAsLin2 (28). Gene families belonging to enriched pathways associated with FMT were extracted by unpacking the reaction components of the pathway.

214 For each enriched pathway, we looked for examples of gene families that were not present within recipients at baseline, but were present 6-weeks post-treatment, and were also present in any one of the contributing donors. To be confident in calling gene presence, the species genome it belonged to was required to be present within a sample at sufficient coverage, such that its absence could not be 
explained by incomplete genome representation. Inspecting the number of genes for a given species within a sample by the median counts per million (CPM) of those genes enabled us to select a conservative universal genome coverage threshold (median CPM $>4$ ), above which we could confidently call gene presence.

\section{RESULTS}

\section{Overview of the Gut Bugs Trial}

The Gut Bugs Trial was a double-blinded, randomized-controlled FMT trial that recruited 87 adolescents with obesity (Fig. 1A). FMT recipients received a total of 28 capsules containing $7 \mathrm{~g}$ of concentrated fecal microbiota derived from four sex-matched lean donors ( $1.75 \mathrm{~g}$ microbiota per donor). Placebo recipients received 28 capsules containing a saline solution. All recipients underwent a bowel cleanse the day before treatment. Capsules were ingested across two clinic sessions, one day apart. Clinical assessments and stool samples were collected at baseline (before the bowel cleanse) and at 6-, 12-, and 26-weeks post-treatment. FMT did not lead to a reduction in body weight or BMI. However, FMT recipients exhibited a reduction in android-to-gynoid fat ratio at all post-treatment time points, an effect that was more marked among females. Furthermore, FMT resulted in a 4.5 fold reduction in the prevalence of metabolic syndrome at week 26 (for full trial results see Leong et al., in submission).

\section{Multi-donor FMT altered gut microbiome composition long-term}

A total of 381 stool samples from recipients and donors were analysed by shotgun metagenomic sequencing at a mean sequencing depth of 23 million reads/sample. To assess the impact of FMT on gut microbiome composition, we generated a Bray-Curtis dissimilarity matrix for all fecal metagenome samples and performed a series of cross-sectional PERMANOVA tests. After adjusting for known microbiome confounders (i.e. age, sex, ethnicity, and sequence batch), treatment group was found to have a significant effect on the gut microbiome composition at week 6 and week 12 accounting for $2.73 \%$ and $2.94 \%$ of the variance, respectively (FDR corrected $q<0.1$, Suppl. Table 1 ). This shift in overall community composition was also accompanied by a temporary increase in alpha diversity at week 6 , specifically within female FMT recipients (Wilcoxon signed-rank test, $p=0.0043$, Suppl. Fig. 

week 12 (Wilcoxon signed-rank test, $p=0.014$, Suppl. Fig. 1). Across all post-treatment time points, both male and female FMT recipients had a significantly higher dissimilarity to their baseline sample compared to placebo recipients (Wilcoxon rank-sum test, $p<0.005$ ). This indicated that a one-off dose of FMT capsules was capable of inducing sustained changes in the gut microbiome for up to 26 weeks post-treatment, above the spontaneous drift observed over the same time period within placebo recipients.

\section{Gut microbiomes of female FMT recipients clustered around one particular donor}

To visualize the variation in gut microbiome composition, we performed multidimensional scaling (MDS) on the species-level Bray-Curtis dissimilarities. Stratified by sex and time point, we observed distinct patterns of microbiome shift in response to FMT (Fig. 1B). Strikingly, the gut microbiomes of female FMT recipients clustered around just one of the four contributing donors, particularly at week 6 . By contrast, the gut microbiomes of male FMT recipients did not appear to move towards one particular donor. However, we did observe a slight clustering of male FMT recipient samples, suggesting their microbiomes had become more similar to one another post-FMT.

After adjusting for baseline similarity, we confirmed that the gut microbiome of female FMT recipients became more similar to female donor DF16 (Fig. 1C). The shift towards DF16 among FMT recipients was observed at week 6 and week 26, but not at week 12 (Wilcoxon rank-sum test; week $6, p=0.016$; week 12, $p=0.27$; week 26, $p=0.0031$, Suppl. Table 2). Within male FMT recipients, there was a subtle shift towards male donor DM08, at week 12 and week 26 (Wilcoxon rank-sum test; week 12, p = 0.059; week 26, $p=0.044$, Suppl. Table 2).

DF16 had the second highest alpha diversity among the female donors, while DM08 was the most diverse male donor (Fig. 1D). There was a positive correlation between the degree of microbiome shift towards a donor and the donors' alpha diversity (Spearman's correlation, $\rho=0.25, p=0.0013$ ). 


\section{FMT transitioned gut microbiomes from Bacteroides to Prevotella dominance}

273 Recent studies have suggested individuals can be stratified into microbial enterotypes according to the 274 ratio of Prevotella to Bacteroides (P/B ratio) $(37,38)$. To investigate whether the observed shifts towards 275 DF16 could be explained by a transition in the P/B ratio, we compared the P/B ratio of donors to those 276 of recipients before and after treatment. As in previous studies $(5,39)$, we defined P/B ratios above 0.1 277 as being high. Female donor DF16 and male donor DM08 were the only donors with a high P/B ratio 278 (Fig. 1E). At baseline, recipients displayed variable P/B ratios, with some recipients characterized by 279 high levels of Prevotella, and others by high levels of Bacteroides (Fig. 1F). For placebo recipients, the $280 \mathrm{P} / \mathrm{B}$ ratio distribution did not change during the 6-month trial, consistent with the individual's enterotypes 281 remaining stable. By contrast, among the FMT recipients, almost all individuals with a low P/B ratio at 282 baseline transitioned to a high $\mathrm{P} / \mathrm{B}$ ratio following FMT (week 6; Wilcoxon signed-rank test, $\mathrm{p}=0.0011$ ). 283 This shift from Bacteroides to Prevotella dominance was largely maintained by FMT recipients out to 28426 weeks post-treatment (week 26; Wilcoxon signed-rank test, $p<0.001$, Fig. 1F). 

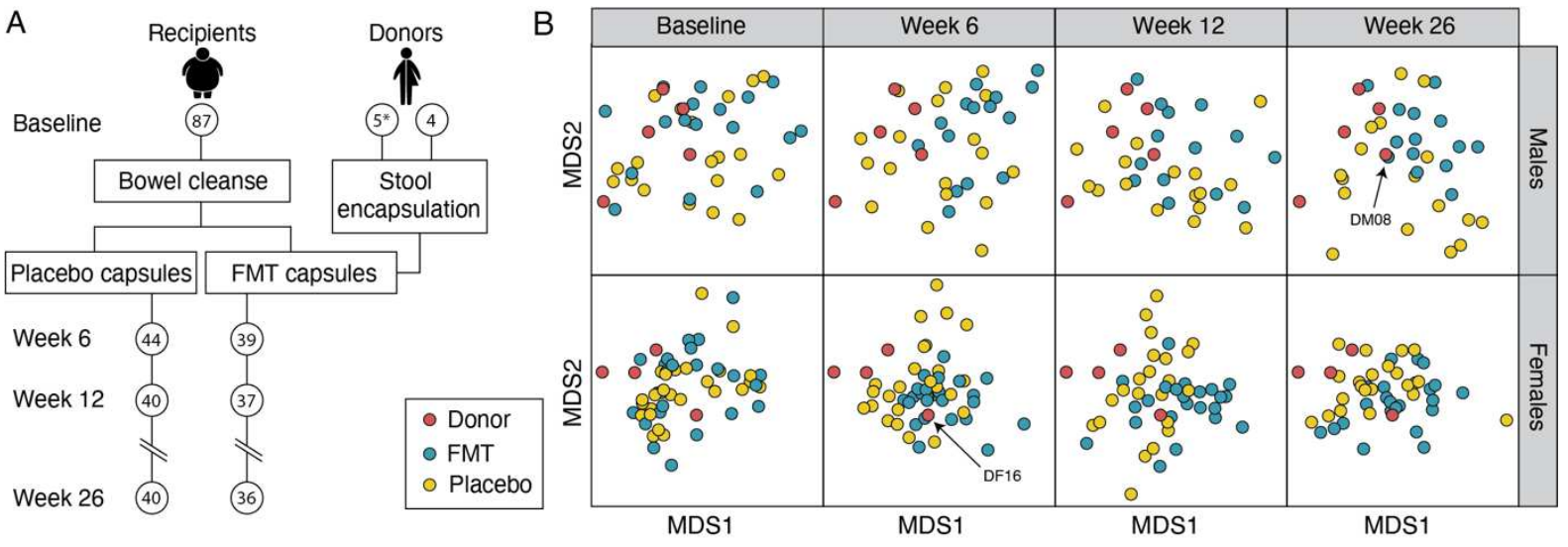

C
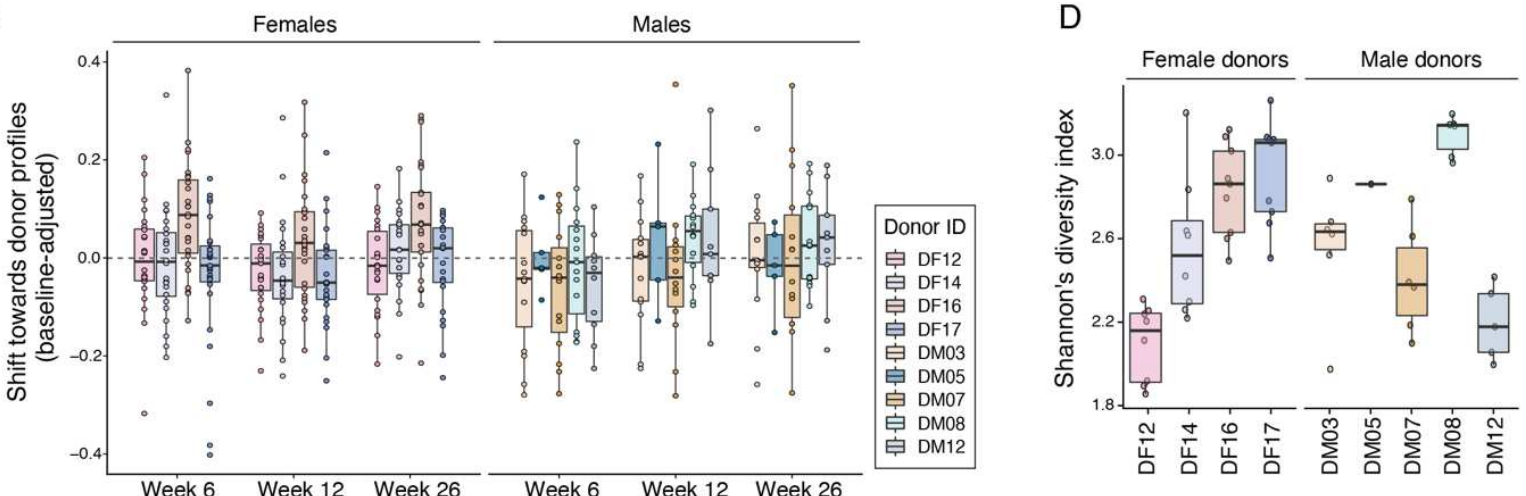

$\mathrm{E}$

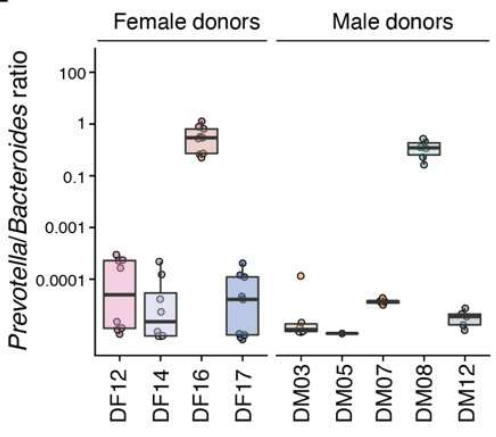

$\mathrm{F}$

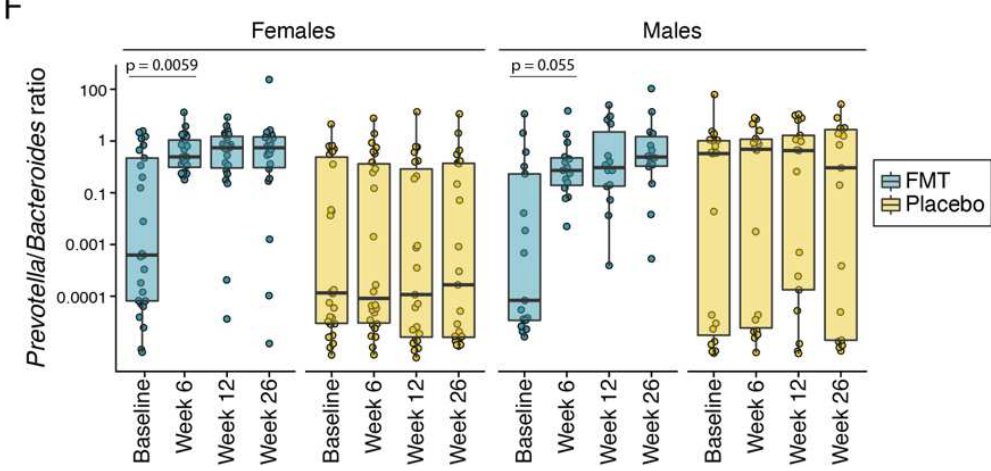

Fig 1. FMT led to prominent shifts in the gut microbiome composition towards particular donors.

288 A. Design of the Gut Bugs Trial. Circles represent stool sample collection time points with corresponding participant numbers indicated. *One male donor was replaced during the trial, hence 5 male donors were recruited. B. Multidimensional scaling plots based on species-level Bray-Curtis dissimilarities, subset by sex and surveyed time point. Multiple samples from each donor, corresponding to each donation batch, were averaged to generate a composite donor profile. C. Shifts in similarity of FMT recipients' fecal metagenome to each contributing donor after adjusting for baseline similarity. D. Alpha diversity of the gut microbiome of donors as measured by Shannon's diversity index. Multiple points correspond to separate donations. E-F. Prevotella/Bacteroides ratio of the gut microbiome of donors 

Wilcoxon signed-rank test.

\section{Dominant donor contributed to species enrichment post-FMT}

The shift towards DF16 among female FMT recipients, and the more subtle shift towards DM08 among males may be partially explained by the $\mathrm{P} / \mathrm{B}$ ratio transition. However, to gain a broader perspective of donor-dependent effects, we surveyed all detectable species to identify those whose relative abundance was significantly altered post-treatment. Using generalized liner modelling as implemented in MaAsLin2, we identified 64 bacterial species that were differentially abundant between baseline and post-treatment time points in FMT recipients (FDR adjusted $q<0.1$ ). Of these, 39 increased and 25 decreased in relative abundance (Fig. 2). The number of differentially abundant species decreased with time; 51 species were altered at week 6, 45 species at week 12, and 34 species at week 26 . The most statistically-significant species enriched included Megamonas hypermegale, Megamonas rupellensis (and unclassified sequences from the genus Megamonas), Bacteroides finegoldii, Bacteroides salyersiae, Bacteroides faecis, Prevotella copri, Desulfovibrio piger, Barnesiella intestinihominis and Ruminococcus lactaris. Species enrichment varied by sex, likely reflecting the difference in donor material. For some species, enrichment could be traced back to one specific donor, predominantly DF16. In fact, many of the species that were unique to, or abundant within DF16, were found to be elevated in female recipients post-FMT (e.g. Desulfovibrio piger, Megamonas rupellensis, Megamonas hypermegale, Megamonas unclassified, Brachyspira unclassified, and Bifidobacterium catenulatum; Suppl. Fig. 2). We also observed a high relative abundance of Prevotella copri in DF16 and DM08 donors when compared to the other donors, which likely contributed to its enrichment among male and female FMT recipients.

To assess whether the shift towards donors was driven by the transfer of novel donor taxa, we removed donor species that were not present in FMT recipients at baseline. Repeating our dissimilarity assessments, we observed that the gut microbiomes of female FMT recipients still shifted in similarity towards DF16 despite the absence of her unique species (Wilcoxon rank-sum test, $p=0.019$ ). This observation is consistent with donor microbiomes providing recipients with novel taxa that fill available niches, while also signalling for wider changes in the endogenous community structure. 
325 Despite a drift in the gut composition of placebo recipients over the course of the study, no individual 326 species were found to be differentially abundant from baseline. This suggests that the species 327 alterations seen within FMT recipients were specific to the FMT treatment, and not caused by the bowel cleanse that preceded treatment, or temporal fluctuations.

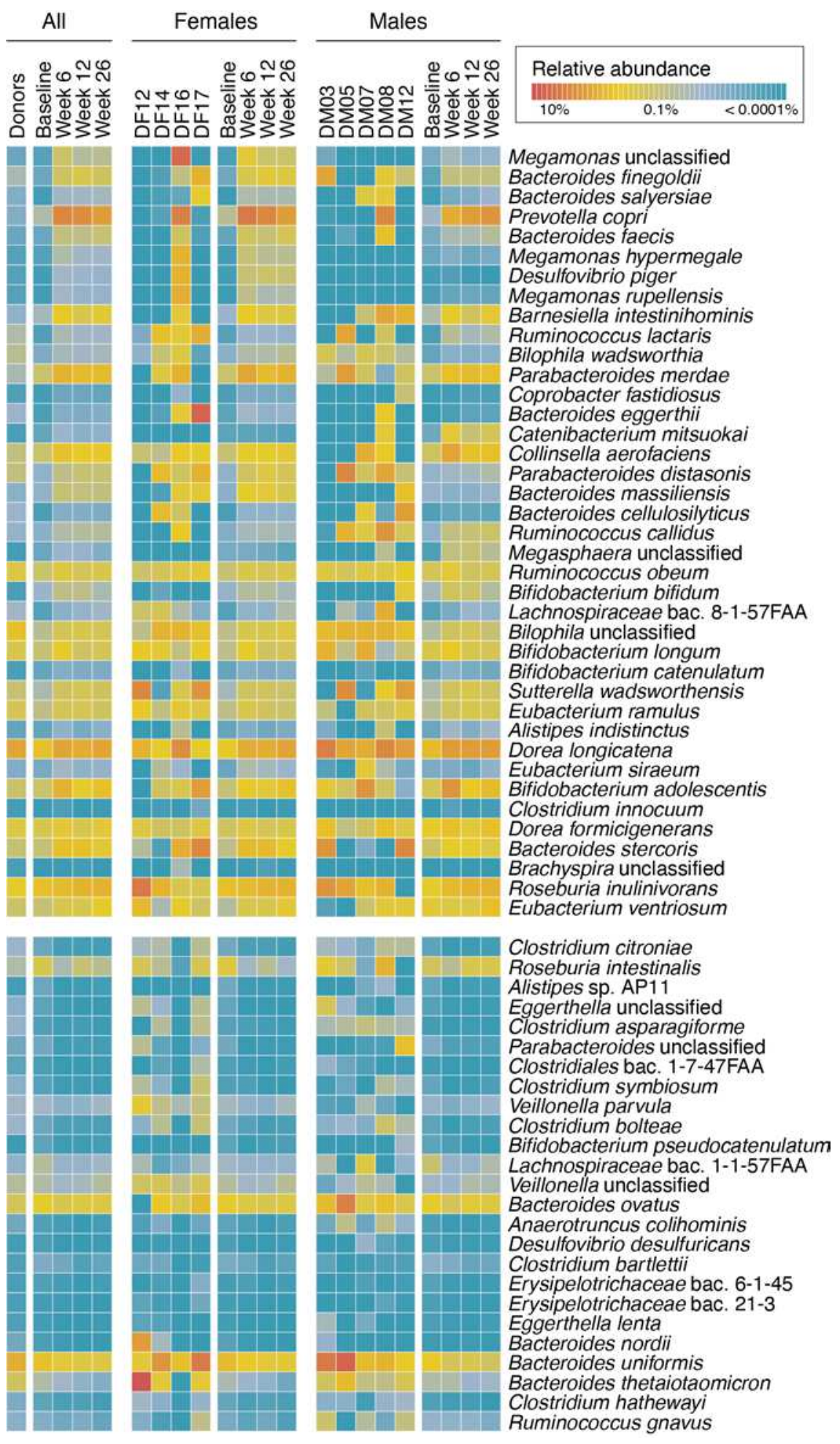


Species are grouped according to whether they were enriched (top panel) or reduced (bottom panel) post-FMT and are listed in order of statistical significance from week 6 onwards (linear model, FDR adjusted $q<0.1)$. Relative abundances were $\log _{10}$-transformed with a small pseudo-count $(1 \mathrm{E}-06)$ added to account for zero abundance values. A relative abundance $<0.0001 \%$ signifies that the species did not pass the minimum threshold abundance level for quantification. Each cell represents the mean transformed relative abundance for a specific species according to the grouping variable; "All" combines male and females averages, while "Females" and "Males" allow species abundances to be subset by sex and contributing donors. Placebo recipient profiles are not displayed, as no bacterial species in their gut microbiome were significantly altered throughout the course of the study.

\section{FMT resulted in durable donor strain engraftment}

Species identification alone cannot always discriminate between different donor sources, nor can it rule out species acquired from the environment. Hence, to gain a more accurate understanding of FMT engraftment from multiple donors, we extended our taxonomic profiling resolution down to the strainlevel. Due to the high level of strain heterogeneity between individuals, finding the same strain from a donor in a post-FMT recipient provides strong evidence of FMT engraftment.

The strain profiling approach we used involved mapping reads against a set of species-specific marker genes to generate a single nucleotide polymorphism (SNP) haplotype representing the dominant strain of a given bacterial species within a sample. The SNP haplotypes were used to construct phylogenetic trees and identify donor strain engraftment events. For example, Bacteroides faecis strains from FMT recipients (post-FMT) were located in close genetic proximity to donor strains, and were clearly separated from their pre-FMT strain (Fig. 3A).

To systematically quantify and monitor strain engraftment events for every species, we selected a genetic strain identity threshold (0.2 median normalized DNA distance), under which strains were considered to be similar enough to constitute a strain match (Fig. 3B). On average, we found that $15 \%$ of the strains in the post-FMT microbiome originated from the donors and were retained for up to 26 weeks post-treatment (Fig. 3C). By comparison, placebo recipients consistently harboured $\leq 1 \%$ donor- 
matching strains. Across both treatment groups, there was a high degree of strain instability reflecting the emergence of novel strains that did not match recipients' baseline strains or any of the donor strains. These novel strains could represent: 1) strains acquired from the environment; 2) donor or recipient strains that were below the detection threshold at baseline; or 3) donor or recipient secondary strains that we were unable to identify. Consistent with the degree of species-level alteration induced by FMT, we found a higher proportion of novel strains among FMT recipients at all post-treatment time points (Pearson's Chi-squared test, $p<2.2 e^{-16}$ ). Collectively, these observation imply that FMT leads to greater fluctuations in strain dominance, which is not solely influenced by donor-engrafting strains, but may also include alterations within the host's endogenous microbiome.

\section{Strain profiling over time revealed distinct patterns of donor engraftment}

By tracking strain dominance over time, we identified a variety of ecological scenarios which included strain retention, strain replacement, strain loss, and strain gain (Fig. 3D). Combining all profiled species, we found the proportions of 6 out of 7 scenarios differed between FMT and placebo recipients. For both groups, we observed relatively high rates of dominant strain replacement from baseline. Replacement by a donor strain only accounted for $11 \%$ of strain replacement events among FMT recipients suggesting a high degree of strain turnover from uncharacterized sources. Donor strain replacement within FMT recipients tended to be stable out to 26 weeks rather than temporary. As expected, placebo recipients were more likely to retain their dominant strain throughout the course of the study indicating a higher degree of microbiome stability.

Among FMT recipients, the most common species in which the dominant strain was replaced was Bacteroides stercoris, whereas recipient strains from Ruminococcus bromii, Bacteroides vulgatus, Eubacterium ventriosum, and Eubacterium rectale remained stable, despite also being present in donors. Recipient strains that were present at lower relative abundance were more likely to be replaced than strains that were at higher relative abundance (Wilcoxon rank-sum test, $p=0.004$, Suppl. Fig. 3A). We also identified cases where recipients had gained a donor strain (either temporarily or long-term) from a species that they did not possess at baseline. This gaining of a new strain occurred more frequently than recipient strain replacement. This result differs from a published observation that conspecific donor strains were more likely to engraft than new species (21). Finally, among FMT 
recipients we discovered a proportionally higher incidence of strain loss without replacement compared to the placebo group. This suggests that the introduction of exogenous strains likely leads to niche replacement and out-competition of both conspecific and heterospecific strains.

A
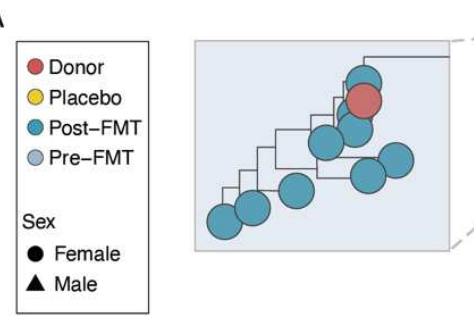

Male

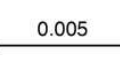

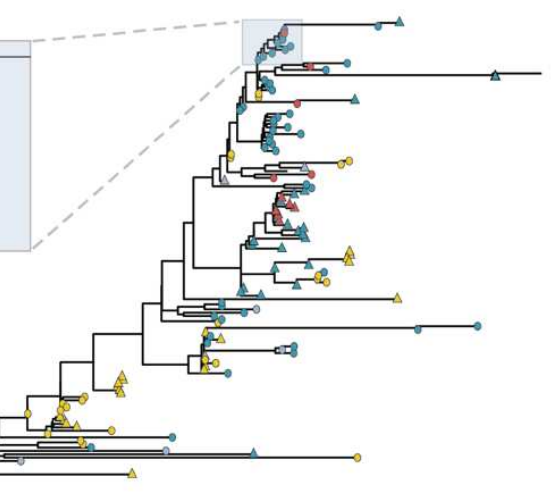

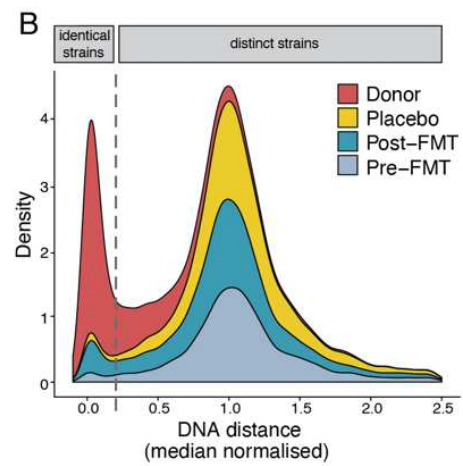

C

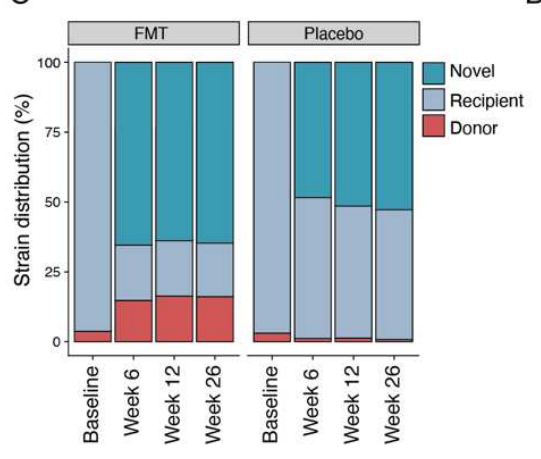

D

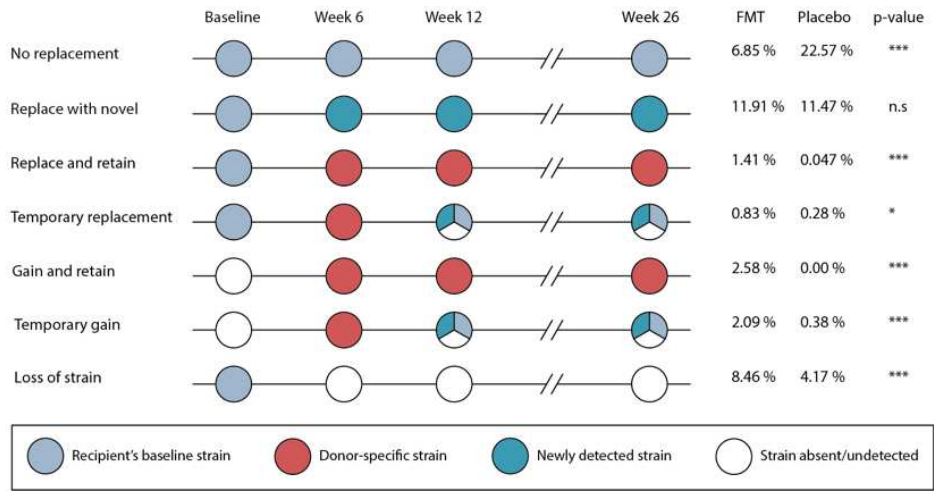

Fig 3. Strain profiling reveals a variety of competition dynamics for conspecific microbial strains.

A. Phylogenetic tree of different Bacteroides faecis strains, one of the species enriched post-FMT. Bacteroides faecis strains were present in $138 \mathrm{fecal}$ metagenomes as determined by SNP haplotyping. Scale bar signifies difference in sequence similarity between SNP haplotypes. B. Distribution of median normalised DNA distances for conspecific strain pairs. Recipient strains (Pre-FMT, Post-FMT, and Placebo) were compared against donor strains from the corresponding treatment batch. Because we had multiple stool samples for each donor, we also compared intra-donor strains (plotted in red). This allowed us to set a universal strain threshold of 0.2 median normalised DNA distance for calling identical strains, as indicated by the vertical dashed line.

C. Proportions of strains identified as being either unique to recipient (matching recipient's baseline strain) or unique to donors (matching any of the 
contributing donor strains). Strains that were newly detected, or that did not match the recipient's baseline strain or any contributing donor strains were designated as "Novel". D. Proportion of longitudinal strain profiling scenarios by treatment group. Differences between FMT and placebo proportions for each scenario were tested by proportion test with significance denoted by ${ }^{*} \mathrm{p}<0.05,{ }^{* * *} \mathrm{p}$ $<0.0005$, n.s. not significant.

\section{FMT recipients exhibited differential degrees of donor strain engraftment}

The degree of donor strain engraftment varied substantially between FMT recipients (Fig. 4A). Whilst we observed a mean donor-strain engraftment proportion of $15 \%$ at week 6 , variation amongst FMT recipients ranged from $0 \%$ through to $60 \%$. There was no difference in the mean proportion of donorstrain engraftment between male and female recipients, with both exhibiting high inter-individual variation (Wilcoxon rank-sum test, $p=0.34$, Suppl. Fig. 3B). Resistance to exogenous donor strain engraftment may reflect the resilience and stability of the endogenous gut community. Microbial diversity is broadly believed to contribute to gut microbiome resilience by providing functional redundancy and colonization resistance (40). Yet, we found no correlation between recipients' Shannon diversity index at baseline and the proportion of donor strain engraftment (Pearson's correlation, $r=$ $0.19, p=0.27$, suggesting other factors are likely involved.

The proportion of overall donor strain engraftment, or that of any one donor, did not correlate with changes in any clinical variables (data not shown). Clinical parameters assessed included anthropometric measures, lipid profile, and markers of glucose metabolism. Thus, in the context of obesity, higher levels of donor engraftment were not associated with improvements in any of the clinical outcomes measured.

\section{Microbially diverse donors contributed more towards strain engraftment}

Donors varied considerably in their engraftment efficiency; that is the proportion of their strains that were observed to engraft within the FMT recipients (Kruskal-Wallis rank sum test, females $p<0.005$, males $p=0.022$ ). Among female donors, DF16, exhibited the highest engraftment efficiency with $4.7 \%$ 
had an average engraftment efficiency of just $0.9 \%$. Among the male donors, DM08 had the highest engraftment efficiency of $4.6 \%$, closely followed by DM12 and DM05 both on $4.4 \%$ (Fig. 4B). These results were largely consistent with the donor-specific contribution of engrafted strains within FMT recipients (Fig. 4C). Among females at week 6, 49\% of donor engrafted strains belonged to DF16, followed by DF14 on $31 \%$, DF17 on $15 \%$, and DF12 on $5 \%$. A strong donor bias was also apparent in the males, with DM08 contributing to $46 \%$ of donor engrafting strains at week 6 , followed by DM12 on 23\%, DM07 on 13\%, DM03 on 10\%, and DM05 on $8 \%$. The proportion of strain engraftment positively correlated with the donors' Shannon diversity index (Pearson's correlation, $r=0.17, p=0.037$ ). Collectively, these strain-level results support the species-level microbiome shifts we observed and provide further evidence that donor microbiomes differ in their engraftment abilities.

A

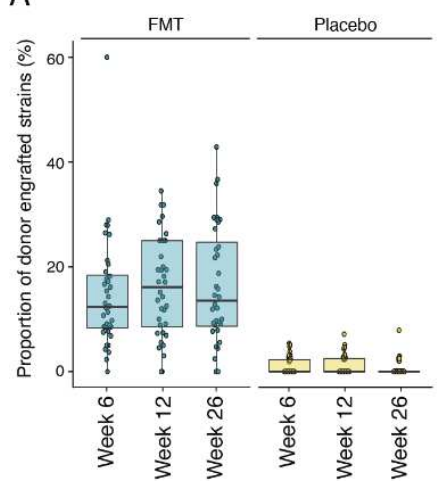

B

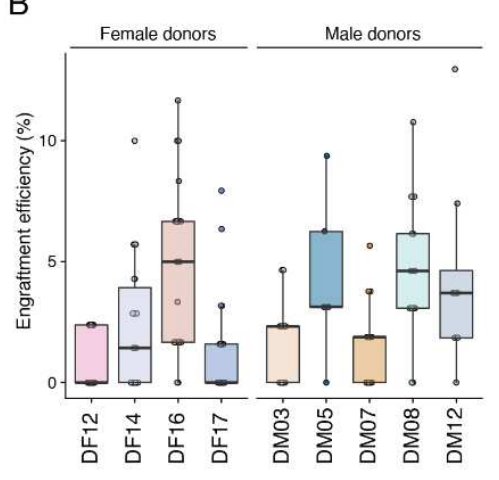

$\mathrm{C}$

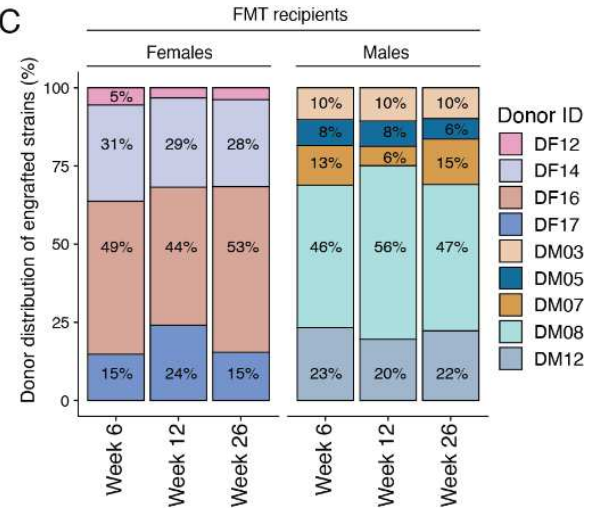

Fig 4. Inter-individual variability in donor strain engraftment.

A. Proportion of donor-engrafted strains in recipients at each post-treatment timepoint. Data points proportion of strains within the donor's fecal metagenome that engrafted among FMT recipients, detected at week 6. C. Donor-specific contributions to overall strain engraftment in FMT recipients.

The therapeutic effect of FMT for the treatment of metabolic disorders may lie in the ability of engrafted 
Therefore, we performed functional profiling using HUMAnN2 (23) to characterize shifts in the functional potential of the microbial gut community in response to FMT.

455 Previous gut microbiome studies have associated obesity and poor metabolic health with low microbial gene richness (41). However, we identified no difference in gene richness between our donors and recipients in our study population at baseline (t-test, $p=0.16$ ). Consistent with the observed variation in alpha diversity (Fig. 1D), we also observed variability in donor gene richness (Suppl. Fig. 4). Female donor DF16 and male donor DM08 exhibited relatively high gene richness in comparison to the other donors, with the exception of male DM07.

Among FMT recipients, we identified an increase in gene richness from baseline at week 6 (Wilcoxon signed-rank test, $p<0.001$ ) and week 26 (Wilcoxon signed-rank test, $p=0.018$ ). This effect was particularly notable within females (Suppl. Fig. 4). To determine the impact of this increase in gene richness on community function, we tested for pathways that were differentially abundant between FMT and placebo recipients at each of the surveyed time points. At baseline, there were no treatment associated pathways. However, at week 6 we identified 10 differentially abundant pathways; 5 that were enriched and 5 that were reduced within FMT recipients (Fig. 5A). The fecal metagenomes of FMT recipients showed a greater potential for nicotinamide adenine dinucleotide (NAD) metabolism, polyamine production, vitamin synthesis (menaquinones and tetrapyyroles), and amino acid metabolism (L-lysine), with concomitant reductions in the potential of a number of pathways, particularly pantothenate and coenzyme A biosynthesis. Subsequent associations at weeks 12 and 26 revealed that the majority of the treatment associated pathways identified at week 6 were not differentially abundant at later time points. Instead, we identified a further 14 pathways with reduced potential among FMT recipients (Suppl. Table 3). These results confirm that a single FMT dose can cause long-lasting alterations to potential microbial community functions.

Of the five pathways enriched by FMT at week 6 , three were specific to females, and two were specific to males. Female FMT recipients were enriched in the potential for NAD biosynthesis from aspartate, polyamine biosynthesis, and 1,4-dihydroxy-6-naphthoate biosynthesis. By contrast, male FMT recipients were enriched for L-lysine biosynthesis and tetrapyrrole biosynthesis from glycine pathways. 
We hypothesized that these sex-specific differences were due to differences in the FMT donor material.

483

484

485

486

487

488

489

490

491

492

493

494

495

496

497

498

499

500

501

502

503

504

505

506

507

508

To test this, we firstly identified the bacterial species that were responsible for pathway enrichment. Across the three female FMT-enriched pathways, we identified 19 contributing species, four of which were shown to be elevated post-FMT; Desulfovibrio piger, Eubacterium siraeum, Megamonas hypermegale, and Megamonas rupellensis. The Megamonas species were unique to donor DF16 and contributed genes to all three female FMT-enriched pathways. Similarly, we identified 20 species responsible for the two male FMT-enriched pathways. Three of these species were elevated post-FMT; Catenibacterium mitsuokai (unique to male donor DM08), Ruminococcus obeum, and Sutterella wadsworthensis.

To determine individual donor contributions to enriched pathways, we tested for genes that had been gained post-FMT that were also present in any of the contributing donors. Across all enriched pathways, we found FMT recipients had gained a higher proportion of these genes compared to placebo recipients, suggesting these had likely been acquired by donor-engrafting species. The majority of the gained genes were traced back to species present within one specific donor. When multiple donors contained the same gene family from the same bacteria, we leveraged dominant strain data to assign the likely donor source. Consistent with these donors having a higher proportion of strain engraftment, we identified the same dominant donors (DF16 and DM08) as contributing proportionately more gene families within the FMT-enriched pathways. For example, female donor DF16 was responsible for the transfer of multiple genes involved in the NAD biosynthesis from aspartate pathway to 18 FMT recipients (Fig. 5B). These genes were present on many of the species identified as being elevated post-FMT, including Prevotella copri, and various species from genus Megamonas. Importantly, the gain of these genes occurred specifically within FMT recipients, ruling out the possibility they were naturally acquired from environmental sources. Our ability to track functionally relevant gene transfer from donor to recipient suggests that FMT-related augmentation is largely dependent on which strains the donor provides and which strains engraft within the recipient. Thus, the observed differences in pathway enrichment between males and females suggest functional alterations induced by FMT are not universal but, rather, specific to the genes present on engrafting strains. 


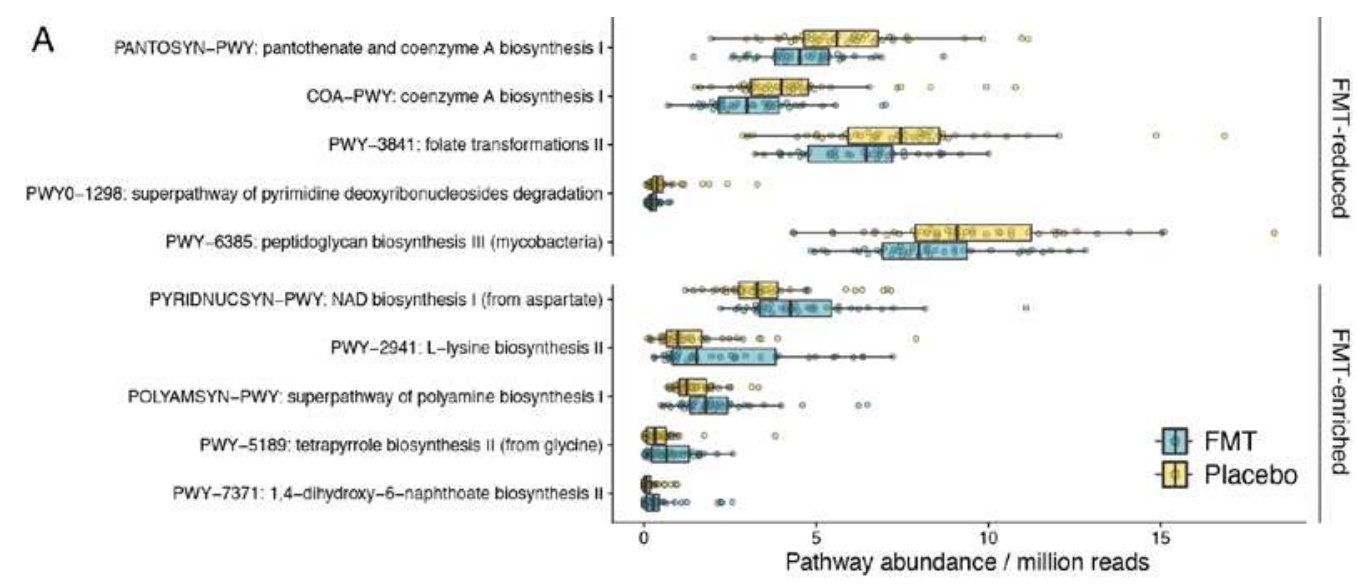

B

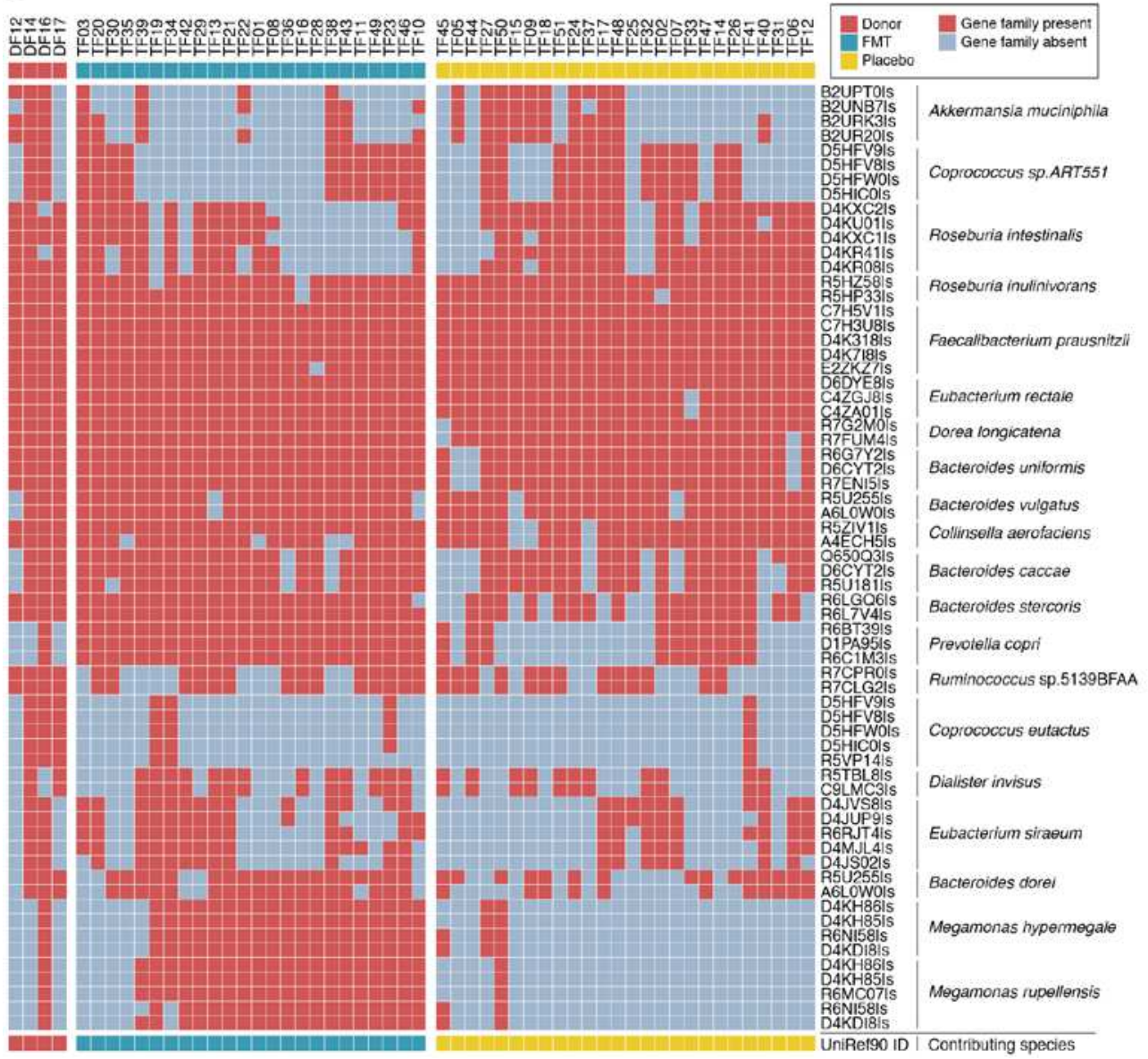

510 Fig 5. FMT-engrafting strains altered the metabolic capacity of the gut microbiome.

511 A. Bacterial metabolic pathways in the gut microbiome found to be differentially abundant between FMT

512 and placebo recipients at week 6 (linear model, FDR adjusted $q<0.2$ ). B. Heatmap displaying UniRef90

513 gene families belonging to the nicotinamide adenine dinucleotide (NAD) biosynthesis from aspartate

514 pathway that were gained (red cells) by female FMT recipients at week 6 (i.e. were not present at

515 baseline). Placebo recipient data were included to differentiate between environmental gain (gene 
516 families likely acquired from common species within the environment) and FMT-specific gain (gene

517 families likely acquired from a donor-engrafting species).

\section{DISCUSSION}

520 Administration of FMT capsules from multiple donors led to sustained alterations in the structure and function of the gut microbiome in adolescents with obesity. In what was in practice a competition experiment between donor microbiomes, we showed that higher levels of strain engraftment occurred for specific bacterial strains from certain donors. The dominant engrafting female and male microbiomes were characterized by high microbial diversity, high gene richness, and a high Prevotella to Bacteroides ratio. FMT with these dominant microbiomes resulted in a stable transition towards a Prevotella dominant enterotype in recipients. We unravelled a variety of strain competition dynamics, including replacement of endogenous strains with conspecific donor strains that persisted within their new hosts for at least 6 months. Finally, we tracked the transfer of genes from donor engrafting strains that led to the enrichment of various metabolic pathways within the recipients' microbial communities.

Previous studies, based on single donor approaches, have shown that bacterial strains from donors engraft with variable success rates $(20,21)$. A larger shift towards the donor, as well as higher levels of FMT engraftment, tends to produce better clinical responses in IBD (11-15). Whilst we acknowledge that the mechanisms responsible for FMT's therapeutic effects may extend beyond bacteria (42-44), the key to successful FMT presumably lies in the selection of desirable donors with high engraftment rates. Such donors can arguably be termed "super-donors" if one considers efficient engraftment a prerequisite for clinical improvement (10). Using a multi-donor approach, we demonstrated that microbiomes from donors with high species diversity and gene richness engraft better than others. This finding is consistent with previous FMT trials for obesity $(5)$ and $\operatorname{IBD}(11,16)$, which also found a positive association between engraftment and microbial diversity of the donor.

540 We contend that female donor DF16 is an example of a super-donor because of her gut microbiomes 541 ability to engraft and augment recipients' local community structure and function. Remarkably, the 542 microbiome composition of all female FMT recipients shifted towards DF16's and retained this structure 543 throughout the 6-month trial. Similar donor-polarizing shifts were not observed in male recipients, 544 despite DM08 contributing towards similar levels of strain engraftment and outcompeting the other male 
donors. We initially hypothesized that the prominent effect of DF16 was due to the transfer of novel taxa that were not present in recipients' microbiomes at baseline. DF16 had a number of novel species that efficiently transferred, including various Megamonas species. Notably, when we removed DF16's novel taxa from our analysis, we observed that the recipients' microbiomes were still more similar to hers than those of the other donors. This suggests that, in addition to providing recipients with novel species, engraftment from DF16's microbiome was also able to alter recipients' endogenous microbial population structure.

We also hypothesized that better engraftment would lead to better clinical response. Yet we found no associations between strain engraftment and clinical improvement across a range of clinical parameters, even when subsetting by donor. These analyses were likely under powered, particularly given the modest clinical responses observed in the trial (Leong et al., 2020). We contend that clinical response to donor engraftment would be better assessed in FMT trials for treatment of more severe forms of dysbiosis (e.g. IBD) where the clinical effect is likely to be more marked.

Recipients were frequently colonized by strains from multiple donors that were largely retained longterm. These results are particularly encouraging given recipients were not asked to change their diet or lifestyle during the course of the study (35). We did, however, observe highly variable levels of engraftment among FMT recipients despite receiving the same dose of donor material. Although the same set of donors were used throughout the study, we cannot rule out the possibility that there may have been slight variations in microbiota composition between capsule batches. Due to the variable nature of stool, this is an inherent limitation with FMT studies. Recipient variability might also reflect compatibility issues with the donors microbiota or higher resilience of the endogenous gut microbiome in some individuals (21). Receptivity to donor strains was not related to the microbial diversity of the recipient at baseline. This suggests that other selection pressures, such as the recipient's diet or immune response, may have played a larger role in determining the colonization success of exogenous donor strains.

570 Prevotella copri was found to consistently transfer and stably colonize FMT recipients. Engraftment of Prevotella copri increased the P/B ratio of FMT recipients, effectively switching the recipients' microbial enterotype. A Prevotella-type signature has previously been shown to be more favourable for weight 
established, and may not be switched simply by consuming more fiber over a 6-month period (38). Our results suggest FMT is a highly effective strategy for switching from a Bacteroides to a Prevotella-type signature. A similar transition from Bacteroides to Prevotella dominance has been reported before in a multi-donor FMT study for ulcerative colitis which involved regular enema-based administration of pooled fecal material from multiple donors (17). Unlike our study design, their stool pooling approach was not standardized across batches, with varying numbers and combinations of donors which prevented them from tracing the engraftment of Prevotella species to any one particular donor. In our study population, this transition was attributed to the presence of just one Prevotella dominant donor within the multi-donor pool. This suggests that Prevotella strains from these donors were able to outcompete the Bacteroides strains from the recipients as well as those from the three other donors.

Interestingly, the Prevotella dominant donors we identified were also the most effective donors for overall strain engraftment. Whether this association was causative or not remains unclear. A similar observation was recently reported by a small FMT pilot trial for obesity where the most effective donor for engraftment was also characterized by a high $\mathrm{P} / \mathrm{B}$ ratio and consistently transferred Prevotella species shifting recipients enterotypes (5). Based on these collective observations, we suggest that future FMT trials for obesity focus on selecting donors with a high P/B ratio, and couple FMT treatment with a high fiber diet. This may result in maximal donor strain engraftment and help transition individuals towards a microbiome profile that is more susceptible to weight loss.

We demonstrated functional shifts in the metabolic potential of the microbial community following FMT. Importantly, we showed that alterations in microbial metabolic pathways were linked to strain engraftment and newly obtained genes from donor microbiomes, particularly those from the dominant donors. Among the altered microbial pathways, we observed an increased biosynthesis potential for a number of bioactive molecules, including NAD, polyamines, and vitamin precursors, which have previously been implicated in metabolic disorders $(46,47)$. NAD, for example, is known to act as an energy sensor and is intricately involved in energy regulation. Low NAD levels are a characteristic of obesity $(47,48)$, and attempts to boost levels by administering NAD precursors improved weight regulation, glycaemic control, and liver function in mice (49-51). FMT may thus represent a novel strategy to increase NAD levels via microbial biosynthesis in the gut. However, because NAD levels were not measured in our study, we cannot confirm whether enrichment of this pathway actually led to increased NAD production. Future FMT characterization studies would subsequently benefit from 
monitoring metabolite production to validate genetic associations and improve our mechanistic understanding of microbiota transfer.

606

\section{CONCLUSION}

In conclusion, our study provides further evidence for the existence of FMT super-donors. Dominant engrafting male and female donor microbiomes harboured diverse microbial species and genes, and were characterized by a high P/B ratio. Pre-screening donor microbiomes for these characteristics may help improve donor strain engraftment and elicit greater change in microbial community function. However, donor selection is just one piece of the puzzle. The high variability of strain engraftment among FMT recipients suggests that undetermined host factors still play a significant role in mediating FMT receptivity. Future experiments should focus on identifying the host components that moderate strain engraftment and their interactions with phenotype.

\section{ABBREVIATIONS}

618 BMI, body mass index; CPM, counts per million; FDR, false discovery rate; FMT, fecal microbiota 619 transplantation; IBD, inflammatory bowel disease; NAD, nicotinamide adenine dinucleotide; P/B ratio, Prevotella to Bacteroides ratio; PCR, polymerase chain reaction; PERMANOVA, permutational multivariate analysis of variance; SNP, single nucleotide polymorphism.

622

\section{ETHICS AND CONSENT}

624 This study was approved by the Northern A Health and Disability Ethics Committee of New Zealand on 8th November 2016 (16/NTA/172). All participants (donors and recipients) provided informed consent.

Not applicable. 
631 Post-processed metagenomic sequencing files and accompanying metadata are deposited in NCBl's SRA database (BioProject PRJNA637785). BioBakery output tables (KneadData read count table, MetaPhIAn2 relative abundance table, HUMAnN2 pathway abundance table) and the StrainPhIAn SNP haplotype files are available at Figshare (private link: https://figshare.com/s/faf0040fce23b83d1591).

635

\section{COMPETING INTERESTS}

637 The authors declare that they have no competing interests.

638

639 FUNDING

640 This research was funded by the Rockfield Trust.

\section{AUTHOR CONTRIBUTIONS}

All authors contributed to the study design. KSWL led the clinical investigations and coordinated sample collection. BCW and TNJ prepared capsules and processed samples for metagenomic sequencing. BCW analysed the metagenomic data with critical input from TV, WSC, and JMO. BCW wrote the manuscript which was edited by TV, TNJ, KSWL, JGBD, VC, DMS, KLB, WSC, and JMO. All authors reviewed and approved the final manuscript.

648

\section{ACKNOWLEDGMENTS}

650

We would like to thank all of the donors and recipients who participated in the trial. We also would like to thank Wendy Ranson, Yara Gerber, Kay Yeoman, Christine Creagh, and Janene Biggs for their valuable assistance during the study. 


\section{REFERENCES}

655 1. Jayasinghe TN, Chiavaroli V, Holland DJ, Cutfield WS, O'Sullivan JM. The New Era of Treatment for Obesity and Metabolic Disorders: Evidence and Expectations for Gut Microbiome Transplantation. Front Cell Infect Microbiol [Internet]. 2016 [cited 2018 May 31];6(15). Available from: http://www.ncbi.nlm.nih.gov/pubmed/26925392

2. Ridaura VK, Faith JJ, Rey FE, Cheng J, Duncan AE, Kau AL, et al. Gut Microbiota from Twins Discordant for Obesity Modulate Metabolism in Mice. Science (80- ) [Internet]. 2013 Sep 6 [cited 2018 Aug 6];341(6150):1241214. Available from:

3. Liou AP, Paziuk M, Luevano J-M, Machineni S, Turnbaugh PJ, Kaplan LM. Conserved Shifts in the Gut Microbiota Due to Gastric Bypass Reduce Host Weight and Adiposity. Sci Transl Med [Internet]. 2013 Mar 27 [cited 2019 May 24];5(178):178ra41. Available from: http://stm.sciencemag.org/cgi/doi/10.1126/scitrans/med.3005687

4. Turnbaugh PJ, Ley RE, Mahowald MA, Magrini V, Mardis ER, Gordon JI. An obesityassociated gut microbiome with increased capacity for energy harvest. Nature [Internet]. 2006 Dec 21 [cited 2018 Aug 6];444(7122):1027-131. Available from:

5. Yu EW, Gao L, Stastka P, Cheney MC, Mahabamunuge J, Torres Soto M, et al. Fecal microbiota transplantation for the improvement of metabolism in obesity: The FMT-TRIM

6. Allegretti JR, Kassam Z, Mullish BH, Chiang A, Carrellas M, Hurtado J, et al. Effects of Fecal double-blind placebo-controlled pilot trial. Basu S, editor. PLOS Med [Internet]. 2020 Mar 9 [cited 2020 Jul 2];17(3):e1003051. Available from: https://dx.plos.org/10.1371/journal.pmed.1003051 
Production and Vascular Inflammation in Patients With Metabolic Syndrome. J Am Heart Assoc [Internet]. 2018 Apr 3 [cited 2019 Jul 3];7(7):e008342. Available from: http://www.ncbi.nlm.nih.gov/pubmed/29581220

8. Kootte RS, Levin E, Salojärvi J, Smits LP, Hartstra A V., Udayappan SD, et al. Improvement of Insulin Sensitivity after Lean Donor Feces in Metabolic Syndrome Is Driven by Baseline Intestinal Microbiota Composition. Cell Metab [Internet]. 2017 Oct 3 [cited 2018 Dec 3];26(4):611-619.e6. Available from: http://www.ncbi.nlm.nih.gov/pubmed/28978426

9. Vrieze A, Van Nood E, Holleman F, Salojärvi J, Kootte RS, Bartelsman JFWM, et al. Transfer of Intestinal Microbiota From Lean Donors Increases Insulin Sensitivity in Individuals With Metabolic Syndrome. Gastroenterology [Internet]. 2012 Oct [cited 2018 May 11];143(4):913916.e7. Available from: http://www.ncbi.nlm.nih.gov/pubmed/22728514

10. Wilson BC, Vatanen T, Cutfield WS, O'Sullivan JM. The super-donor phenomenon in fecal microbiota transplantation. Front Cell Infect Microbiol [Internet]. 2019 [cited 2020 Jul 2];9(2). Available from:/pmc/articles/PMC6348388/?report=abstract

11. Vermeire S, Joossens M, Verbeke K, Wang J, Machiels K, Sabino J, et al. Donor Species Richness Determines Faecal Microbiota Transplantation Success in Inflammatory Bowel Disease. J Crohn's Colitis [Internet]. 2016 Apr 1 [cited 2018 May 2];10(4):387-94. Available from: https://academic.oup.com/ecco-jcc/article-lookup/doi/10.1093/ecco-jcc/jjv203

12. Moayyedi P, Surette MG, Kim PT, Libertucci J, Wolfe M, Onischi C, et al. Fecal Microbiota Transplantation Induces Remission in Patients With Active Ulcerative Colitis in a Randomized Controlled Trial. Gastroenterology [Internet]. 2015 Jul 1 [cited 2018 May 3];149(1):102-109.e6. Available from: https://www.sciencedirect.com/science/article/pii/S0016508515004515?via\%3Dihub

13. Rossen NG, Fuentes S, van der Spek MJ, Tijssen JG, Hartman JHA, Duflou A, et al. Findings From a Randomized Controlled Trial of Fecal Transplantation for Patients With Ulcerative Colitis. Gastroenterology [Internet]. 2015 Jul 1 [cited 2018 May 3];149(1):110-118.e4. Available from: https://www.sciencedirect.com/science/article/pii/S0016508515004485?via\%3Dihub 
Microbial Diversity Following Fecal Microbiota Transplant for Active Crohn's Disease. Inflamm Bowel Dis [Internet]. 2016 Sep 1 [cited 2018 May 2];22(9):2182-90. Available from: https://academic.oup.com/ibdjournal/article/22/9/2182-2190/4562012

15. Sokol H, Landman C, Seksik P, Berard L, Montil M, Nion-Larmurier I, et al. Fecal microbiota transplantation to maintain remission in Crohn's disease: A pilot randomized controlled study. Microbiome [Internet]. 2020 Feb 3 [cited 2020 Oct 1];8(1):12. Available from: https://microbiomejournal.biomedcentral.com/articles/10.1186/s40168-020-0792-5

16. Kump P, Wurm P, Gröchenig HP, Wenzl H, Petritsch W, Halwachs B, et al. The taxonomic composition of the donor intestinal microbiota is a major factor influencing the efficacy of faecal microbiota transplantation in therapy refractory ulcerative colitis. Aliment Pharmacol Ther [Internet]. 2018 Jan [cited 2018 Jul 2];47(1):67-77. Available from: http://www.ncbi.nlm.nih.gov/pubmed/29052237

17. Paramsothy S, Kamm MA, Kaakoush NO, Walsh AJ, van den Bogaerde J, Samuel D, et al. Multidonor intensive faecal microbiota transplantation for active ulcerative colitis: a randomised placebo-controlled trial. Lancet [Internet]. 2017 Mar 25 [cited 2018 May 21];389(10075):121828. Available from: http://www.ncbi.nlm.nih.gov/pubmed/28214091

18. Bajaj JS, Kassam Z, Fagan A, Gavis EA, Liu E, Cox IJ, et al. Fecal microbiota transplant from a rational stool donor improves hepatic encephalopathy: A randomized clinical trial. Hepatology [Internet]. 2017 Dec [cited 2018 Aug 6];66(6):1727-38. Available from: http://www.ncbi.nlm.nih.gov/pubmed/28586116

19. Kazerouni A, Wein LM. Exploring the Efficacy of Pooled Stools in Fecal Microbiota Transplantation for Microbiota-Associated Chronic Diseases. PLoS One [Internet]. 2017 [cited 2018 Sep 4];12(1):e0163956. Available from: http://www.ncbi.nlm.nih.gov/pubmed/28068341

20. Smillie CS, Sauk J, Gevers D, Friedman J, Sung J, Youngster I, et al. Strain Tracking Reveals the Determinants of Bacterial Engraftment in the Human Gut Following Fecal Microbiota Transplantation. Cell Host Microbe [Internet]. 2018 Feb 14 [cited 2018 May 2];23(2):229240.e5. Available from: https://www.sciencedirect.com/science/article/pii/S1931312818300386

21. Li SS, Zhu A, Benes V, Costea PI, Hercog R, Hildebrand F, et al. Durable coexistence of 
donor and recipient strains after fecal microbiota transplantation. Science (80- ) [Internet]. 2016 Apr 29 [cited 2018 May 24];352(6285):586-9. Available from: http://www.ncbi.nlm.nih.gov/pubmed/27126044

22. Truong DT, Franzosa EA, Tickle TL, Scholz M, Weingart G, Pasolli E, et al. MetaPhIAn2 for enhanced metagenomic taxonomic profiling [Internet]. Vol. 12, Nature Methods. Nature Publishing Group; 2015 [cited 2020 Jun 29]. p. 902-3. Available from: https://www.nature.com/articles/nmeth.3589

23. Franzosa EA, Mclver LJ, Rahnavard G, Thompson LR, Schirmer M, Weingart G, et al. Species-level functional profiling of metagenomes and metatranscriptomes. Nat Methods [Internet]. 2018 Nov 30 [cited 2019 May 30];15(11):962-8. Available from: http://www.nature.com/articles/s41592-018-0176-y

24. Truong DT, Tett A, Pasolli E, Huttenhower C, Segata N. Microbial strain-level population structure \& genetic diversity from metagenomes. Genome Res. 2017 Apr 1;27(4):626-38.

25. Wickham H, Averick M, Bryan J, Chang W, D'Agostino McGowan L, François R, et al. Welcome to the tidyverse. J Open Source Softw [Internet]. 2019;4(43):1686. Available from: https://doi.org/10.21105/joss.01686

26. Vatanen T, Plichta DR, Somani J, Münch PC, Arthur TD, Hall AB, et al. Genomic variation and strain-specific functional adaptation in the human gut microbiome during early life. Nat Microbiol [Internet]. 2019 Mar 1 [cited 2020 Jul 2];4(3):470-9. Available from: https://pubmed.ncbi.nlm.nih.gov/30559407/

27. Oksanen J, Blanchet FG, Friendly M, Kindt R, Legendre P, McGlinn D, et al. vegan: Community Ecology Package. R package version 2.5-6. [Internet]. 2019. Available from: https://cran.r-project.org/package=vegan

28. Mallick H, Rahnavard A, Mclver L. Maaslin2. R package version 0.99.18. [Internet]. 2019. Available from: http://huttenhower.sph.harvard.edu/maaslin2\%0D

29. Kolde R. pheatmap: Pretty Heatmaps. R package version 1.0.12. 2019.

30. Schliep KP. phangorn: Phylogenetic analysis in R. Bioinformatics [Internet]. 2011 Feb 15 [cited 
2020 Sep 24];27(4):592-3. Available from:

https://academic.oup.com/bioinformatics/article/27/4/592/198887

31. Yu G, Smith DK, Zhu H, Guan Y, Lam TTY. ggtree: an r package for visualization and annotation of phylogenetic trees with their covariates and other associated data. Methods Ecol Evol [Internet]. 2017 Jan 1 [cited 2020 Sep 24];8(1):28-36. Available from: https://besjournals.onlinelibrary.wiley.com/doi/full/10.1111/2041-210X.12628

32. Clarke E, Sherrill-Mix S. ggbeeswarm: Categorical Scatter (Violin Point) Plots. R package version 0.6.0. [Internet]. 2017. Available from: https://cran.r-project.org/package=ggbeeswarm

33. Wilke CO. cowplot: Streamlined Plot Theme and Plot Annotations for "ggplot2". R package version 1.0.0. [Internet]. 2019. Available from: https://cran.r-project.org/package=cowplot

34. Ram K, Wickham H. wesanderson: A Wes Anderson Palette Generator. R package version 0.3.6. [Internet]. 2018. Available from: https://cran.r-project.org/package=wesanderson

35. Leong KSW, Jayasinghe TN, Derraik JGB, Albert BB, Chiavaroli V, Svirskis DM, et al. Protocol for the Gut Bugs Trial: A randomised double-blind placebo-controlled trial of gut microbiome transfer for the treatment of obesity in adolescents. BMJ Open [Internet]. 2019 Apr 1 [cited 2020 Jul 2];9(4):e026174. Available from: https://pubmed.ncbi.nlm.nih.gov/31005929/

36. Schliep K, Potts AJ, Morrison DA, Grimm GW. Intertwining phylogenetic trees and networks. Fitzjohn R, editor. Methods Ecol Evol [Internet]. 2017 Oct 12 [cited 2020 Sep 24];8(10):121220. Available from: https://onlinelibrary.wiley.com/doi/abs/10.1111/2041-210X.12760

37. Costea PI, Hildebrand F, Arumugam M, Bäckhed F, Blaser MJ, Bushman FD, et al. Enterotypes in the landscape of gut microbial community composition. Nat Microbiol [Internet]. 2018 Jan 18 [cited 2018 May 29];3(1):8-16. Available from: http://www.ncbi.nlm.nih.gov/pubmed/29255284

38. Roager HM, Licht TR, Poulsen SK, Larsen TM, Bahl MI. Microbial enterotypes, inferred by the Prevotella-to-Bacteroides ratio, remained stable during a 6-month randomized controlled diet intervention with the new nordic diet. Appl Environ Microbiol [Internet]. 2014 Feb [cited 2020 Jul 10];80(3):1142-9. Available from: /pmc/articles/PMC3911217/?report=abstract 
39. Hjorth MF, Roager HM, Larsen TM, Poulsen SK, Licht TR, Bahl MI, et al. Pre-treatment microbial Prevotella-to-Bacteroides ratio, determines body fat loss success during a 6-month randomized controlled diet intervention. Int J Obes [Internet]. 2018 Mar 1 [cited 2020 Jul 10];42(3):580-3. Available from: https://pubmed.ncbi.nlm.nih.gov/28883543/

40. Lozupone CA, Stombaugh JI, Gordon JI, Jansson JK, Knight R. Diversity, stability and resilience of the human gut microbiota. Vol. 489, Nature. NIH Public Access; 2012. p. 220-30.

41. Le Chatelier E, Nielsen T, Qin J, Prifti E, Hildebrand F, Falony G, et al. Richness of human gut microbiome correlates with metabolic markers. Nature [Internet]. 2013 Aug 29 [cited 2018 May 31];500(7464):541-6. Available from: http://www.ncbi.nlm.nih.gov/pubmed/23985870

42. Zuo T, Wong SH, Lam K, Lui R, Cheung K, Tang W, et al. Bacteriophage transfer during faecal microbiota transplantation in Clostridium difficile infection is associated with treatment outcome. Gut [Internet]. 2018 May 24 [cited 2018 Dec 3];67(4):634-43. Available from: http://www.ncbi.nlm.nih.gov/pubmed/28539351

43. Ott SJ, Waetzig GH, Rehman A, Moltzau-Anderson J, Bharti R, Grasis JA, et al. Efficacy of Sterile Fecal Filtrate Transfer for Treating Patients With Clostridium difficile Infection. Gastroenterology [Internet]. 2017 Mar [cited 2018 Dec 3];152(4):799-811.e7. Available from: http://www.ncbi.nlm.nih.gov/pubmed/27866880

44. Conceição-Neto N, Deboutte W, Dierckx T, Machiels K, Wang J, Yinda KC, et al. Low eukaryotic viral richness is associated with faecal microbiota transplantation success in patients with UC. Gut [Internet]. 2018 Aug [cited 2018 Dec 3];67(8):1558-9. Available from: http://www.ncbi.nlm.nih.gov/pubmed/29066574

45. Hjorth MF, Blædel T, Bendtsen LQ, Lorenzen JK, Holm JB, Kiilerich P, et al. Prevotella-toBacteroides ratio predicts body weight and fat loss success on 24-week diets varying in macronutrient composition and dietary fiber: results from a post-hoc analysis. Int J Obes. 2019 Jan 1;43(1):149-57.

46. Ramos-Molina B, Queipo-Ortuño MI, Lambertos A, Tinahones FJ, Peñafiel R. Dietary and gut microbiota polyamines in obesity- And age-related diseases. Front Nutr [Internet]. 2019 Mar 14 [cited 2020 Jul 10];6(24). Available from: www.frontiersin.org 
47. Okabe K, Yaku K, Tobe K, Nakagawa T. Implications of altered NAD metabolism in metabolic disorders. J Biomed Sci [Internet]. 2019 May 11 [cited 2020 Jul 10];26(1):24. Available from: /pmc/articles/PMC6511662/?report=abstract

48. Jukarainen S, Heinonen S, Rämö JT, Rinnankoski-Tuikka R, Rappou E, Tummers M, et al. Obesity is associated with low nad+/sirt pathway expression in adipose tissue of BMIdiscordant monozygotic twins. J Clin Endocrinol Metab [Internet]. 2016 Jan 1 [cited 2020 Jul 10];101(1):275-83. Available from: https://pubmed.ncbi.nlm.nih.gov/26574954/

49. Trammell SAJ, Weidemann BJ, Chadda A, Yorek MS, Holmes A, Coppey LJ, et al. Nicotinamide riboside opposes type 2 diabetes and neuropathy in mice. Sci Rep [Internet]. 2016 May 27 [cited 2020 Jul 10];6(26933). Available from: https://pubmed.ncbi.nlm.nih.gov/27230286/

50. Cantó C, Houtkooper RH, Pirinen E, Youn DY, Oosterveer MH, Cen Y, et al. The NAD+ precursor nicotinamide riboside enhances oxidative metabolism and protects against high-fat diet-induced obesity. Cell Metab [Internet]. 2012 Jun 6 [cited 2020 Jul 10];15(6):838-47. Available from: https://pubmed.ncbi.nlm.nih.gov/22682224/

51. Yoshino J, Mills KF, Yoon MJ, Imai SI. Nicotinamide mononucleotide, a key NAD + intermediate, treats the pathophysiology of diet- and age-induced diabetes in mice. Cell Metab [Internet]. 2011 Oct 5 [cited 2020 Jul 10];14(4):528-36. Available from: https://pubmed.ncbi.nlm.nih.gov/21982712/ 
Fig 1. FMT led to prominent shifts in the gut microbiome composition towards particular donors.

849 A. Design of the Gut Bugs Trial. Circles represent stool sample collection time points with corresponding participant numbers indicated. *One male donor was replaced during the trial, hence 5 male donors were recruited. B. Multidimensional scaling plots based on species-level Bray-Curtis dissimilarities, subset by sex and surveyed time point. Multiple samples from each donor, corresponding to each donation batch, were averaged to generate a composite donor profile. C. Shifts in similarity of FMT diversity of the gut microbiome of donors as measured by Shannon's diversity index. Multiple points correspond to separate donations. E-F. Prevotella/Bacteroides ratio of the gut microbiome of donors (E) and FMT and placebo recipients (F). Differences from baseline to week 6 were measured by Wilcoxon signed-rank test.

Fig 2. Bacterial species of the gut microbiome whose relative abundance was altered post-FMT.

Species are grouped according to whether they were enriched (top panel) or reduced (bottom panel) post-FMT and are listed in order of statistical significance from week 6 onwards (linear model, FDR adjusted $q<0.1)$. Relative abundances were $\log _{10}$-transformed with a small pseudo-count $(1 \mathrm{E}-06)$ added to account for zero abundance values. A relative abundance $<0.0001 \%$ signifies that the species did not pass the minimum threshold abundance level for quantification. Each cell represents the mean transformed relative abundance for a specific species according to the grouping variable; "All" combines male and females averages, while "Females" and "Males" allow species abundances to be subset by sex and contributing donors. Placebo recipient profiles are not displayed, as no bacterial species in their gut microbiome were significantly altered throughout the course of the study.

870 strains. 
873 A. Phylogenetic tree of different Bacteroides faecis strains, one of the species enriched post-FMT.

874 Bacteroides faecis strains were present in 138 fecal metagenomes as determined by SNP haplotyping.

875 Scale bar signifies difference in sequence similarity between SNP haplotypes. B. Distribution of median 876 normalised DNA distances for conspecific strain pairs. Recipient strains (Pre-FMT, Post-FMT, and 877 Placebo) were compared against donor strains from the corresponding treatment batch. Because we 878 had multiple stool samples for each donor, we also compared intra-donor strains (plotted in red). This 879 allowed us to set a universal strain threshold of 0.2 median normalised DNA distance for calling identical strains, as indicated by the vertical dashed line. C. Proportions of strains identified as being either unique to recipient (matching recipient's baseline strain) or unique to donors (matching any of the contributing donor strains). Strains that were newly detected, or that did not match the recipient's baseline strain or any contributing donor strains were designated as "Novel".

D. Proportion of longitudinal strain profiling scenarios by treatment group. Differences between FMT and placebo proportions for each scenario were tested by proportion test with significance denoted by ${ }^{*} p<0.05,{ }^{* \star *} p$ $<0.0005$, n.s. not significant.

887

Fig 4. Inter-individual variability in donor strain engraftment.

A. Proportion of donor-engrafted strains in recipients at each post-treatment timepoint. Data points proportion of strains within the donor's fecal metagenome that engrafted among FMT recipients, detected at week 6. C. Donor-specific contributions to overall strain engraftment in FMT recipients.

Fig 5. FMT-engrafting strains altered the metabolic capacity of the gut microbiome.

895 A. Bacterial metabolic pathways in the gut microbiome found to be differentially abundant between FMT and placebo recipients at week 6 (linear model, FDR adjusted q < 0.2). B. Heatmap displaying UniRef90 gene families belonging to the nicotinamide adenine dinucleotide (NAD) biosynthesis from aspartate pathway that were gained (red cells) by female FMT recipients at week 6 (i.e. were not present at baseline). Placebo recipient data were included to differentiate between environmental gain (gene 
900 families likely acquired from common species within the environment) and FMT-specific gain (gene 901 families likely acquired from a donor-engrafting species).

902

903

904

905 
Figures
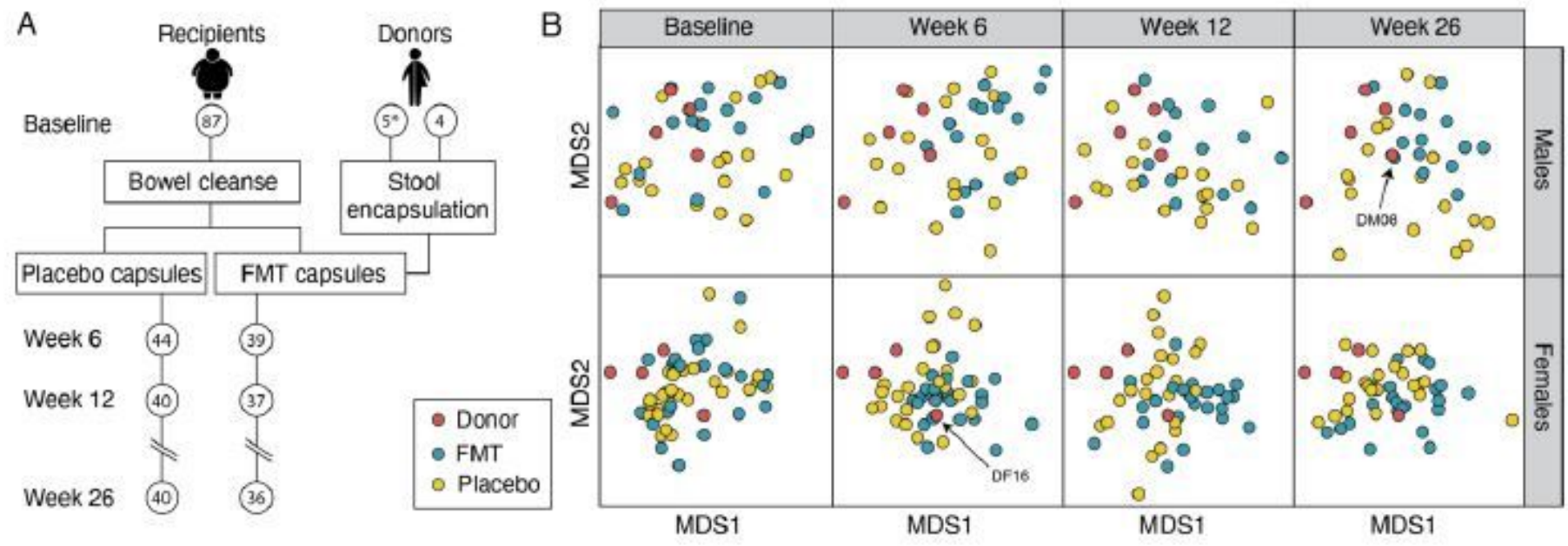

C
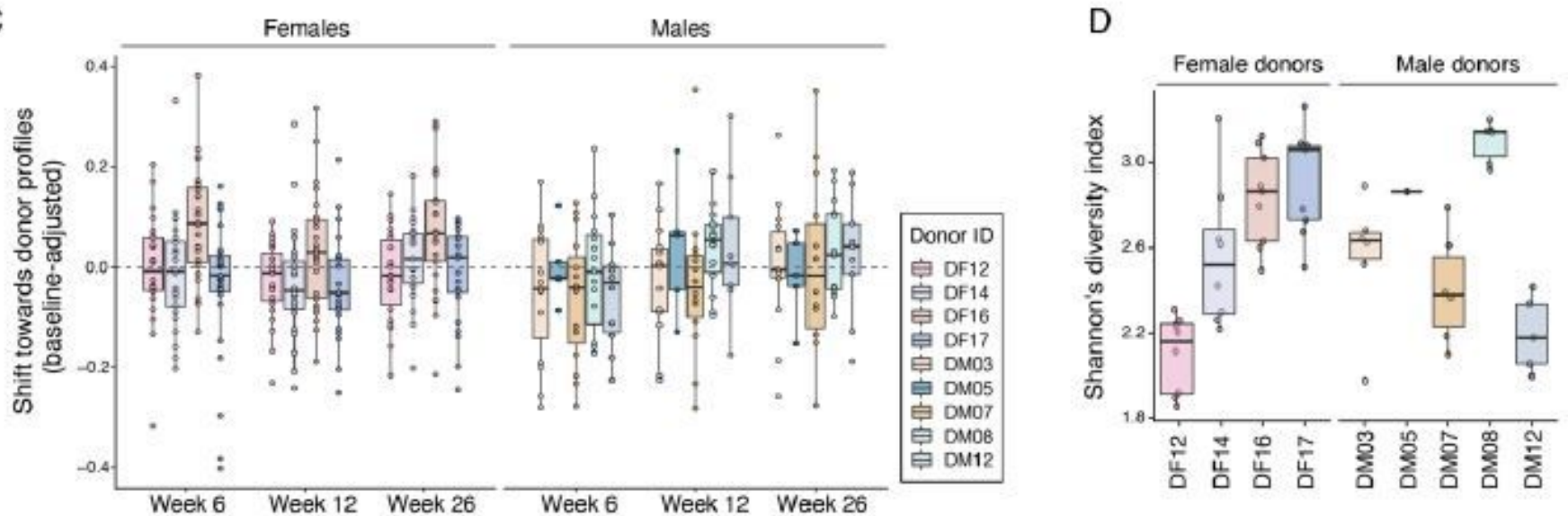

E

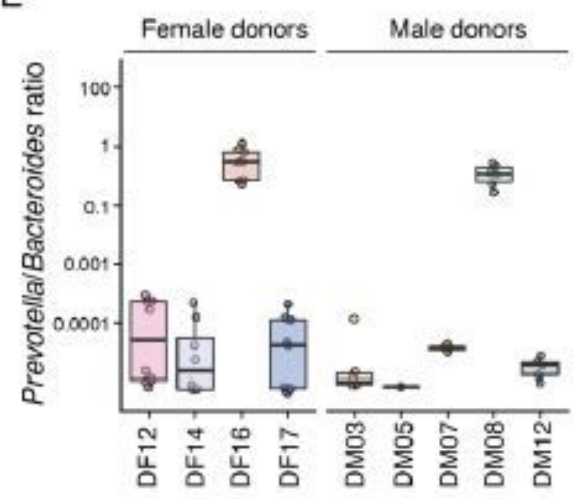

F

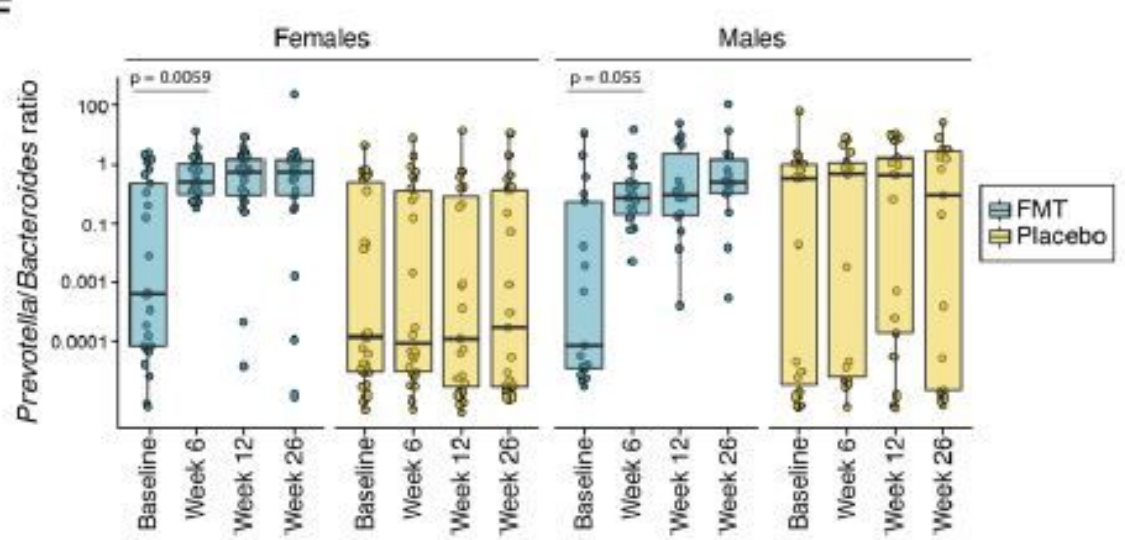

Figure 1

FMT led to prominent shifts in the gut microbiome composition towards particular donors. 


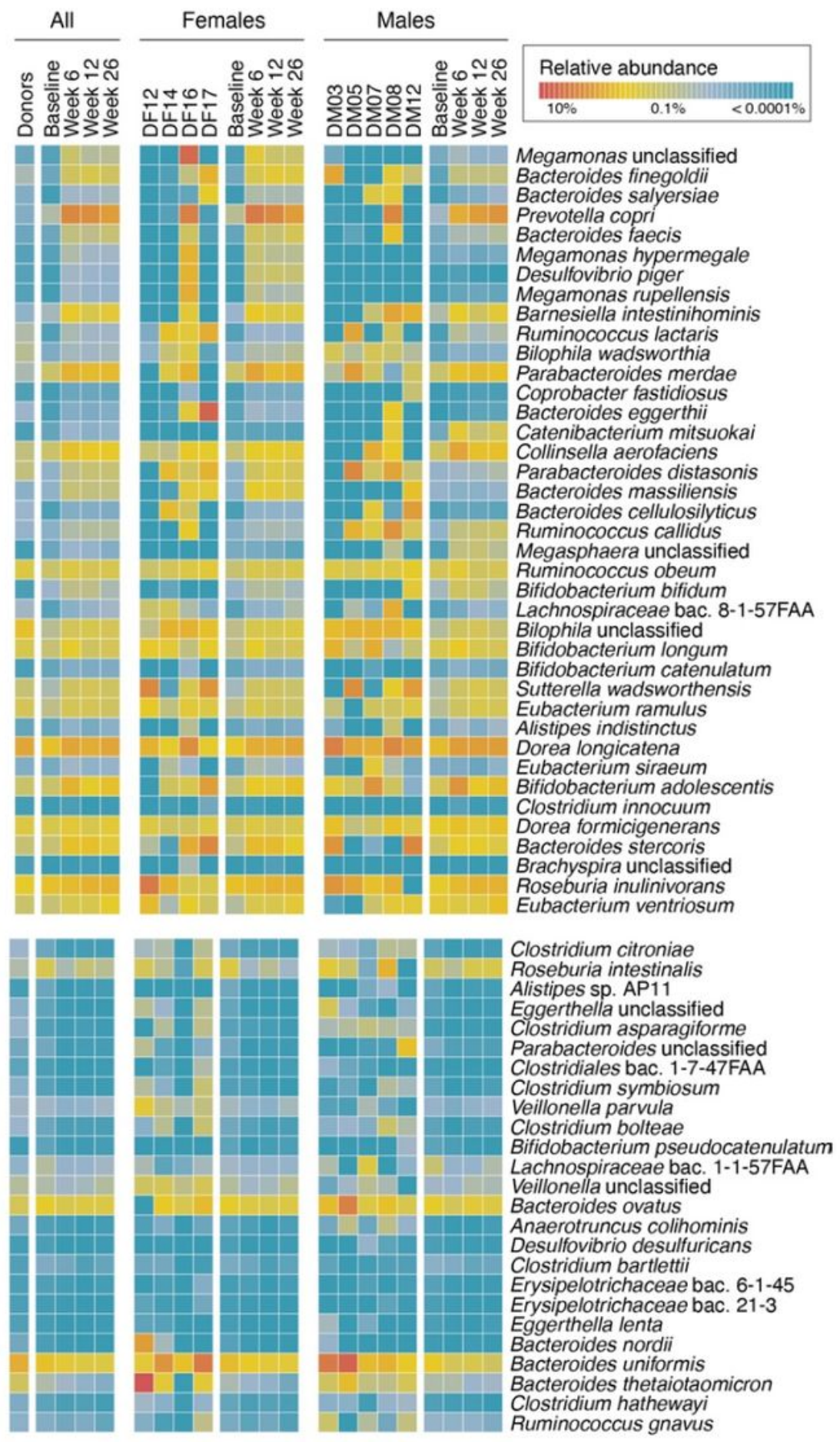

\section{Figure 2}

Bacterial species of the gut microbiome whose relative abundance was altered post-FMT. 
A

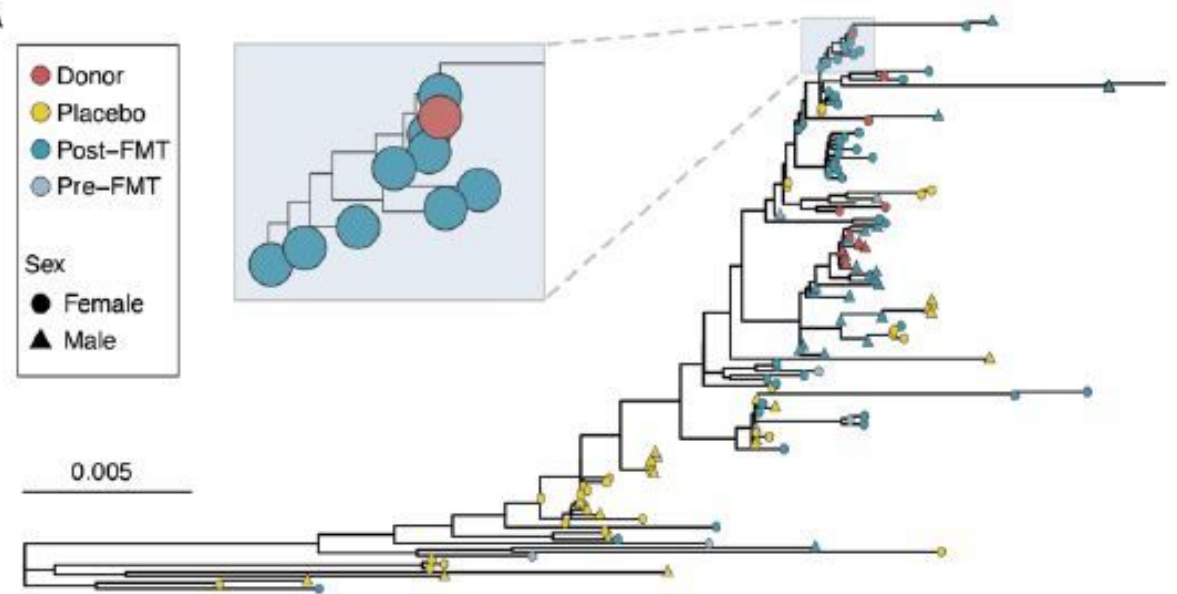

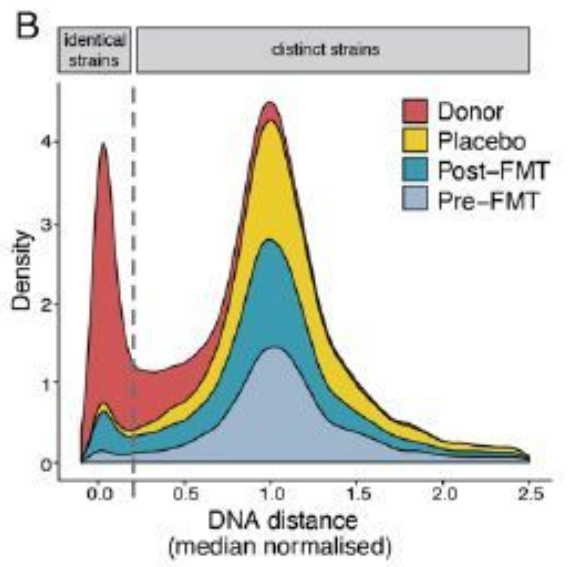
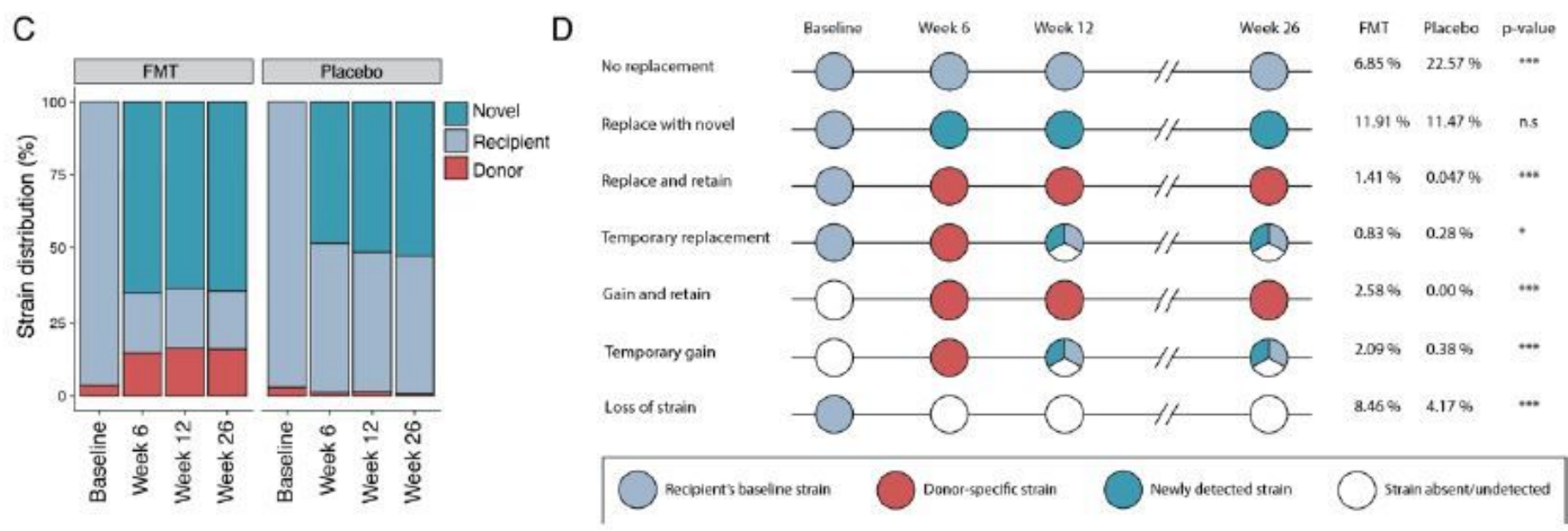

Figure 3

Strain profiling reveals a variety of competition dynamics for conspecific microbial strains.
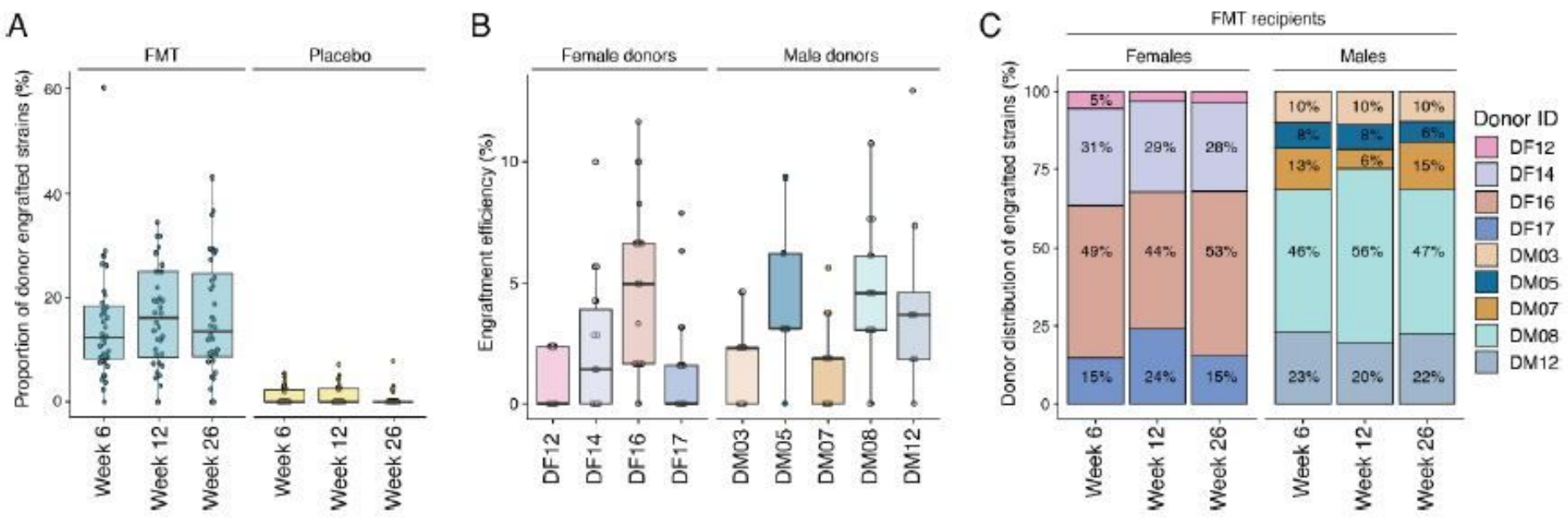

Figure 4

Inter-individual variability in donor strain engraftment. 

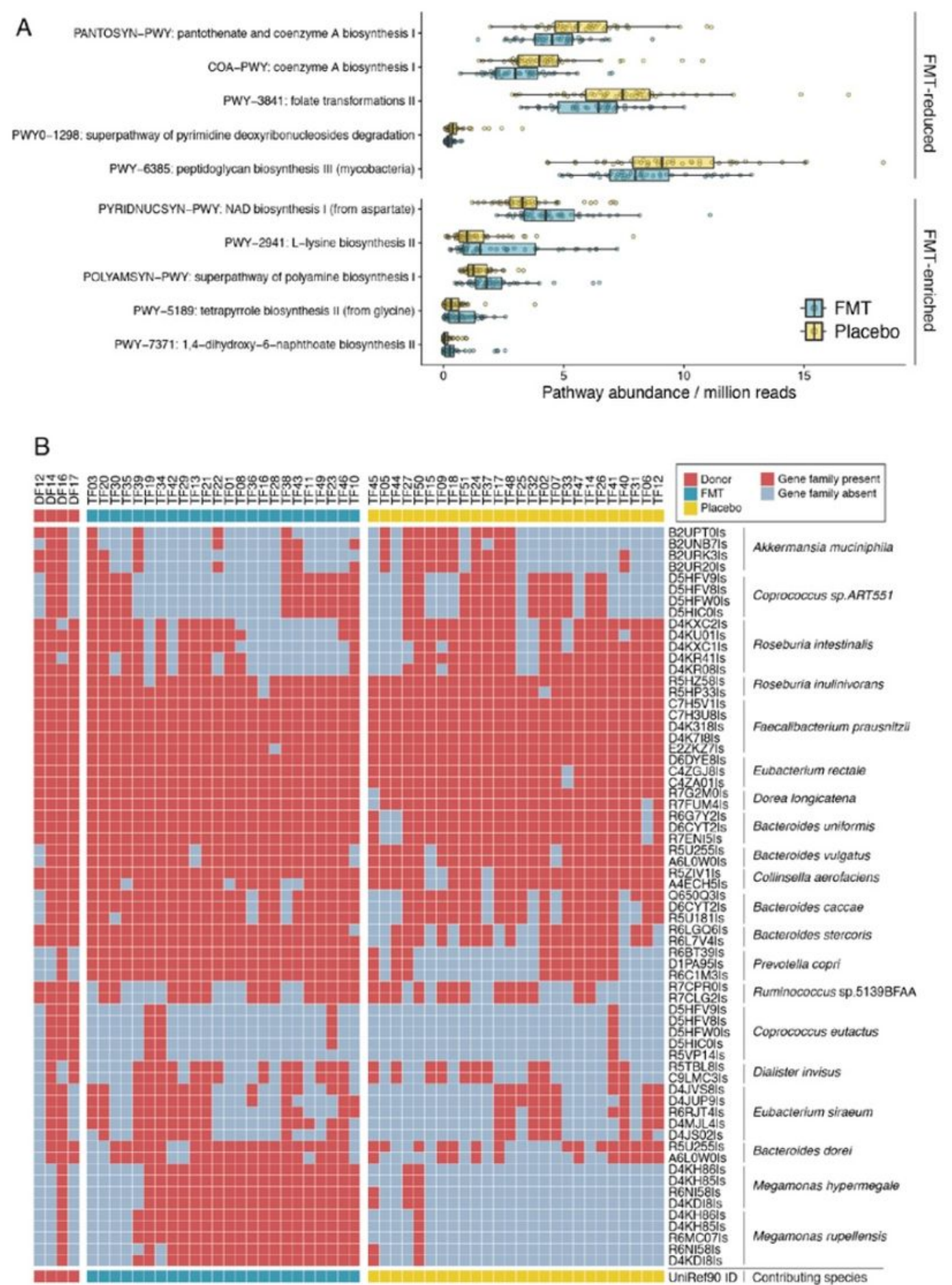

Figure 5

FMT-engrafting strains altered the metabolic capacity of the gut microbiome.

\section{Supplementary Files}

This is a list of supplementary files associated with this preprint. Click to download. 
- microbiomegutbugssupplementarymaterial.pdf 